\title{
A signalome screening approach in the autoinflammatory disease TNF Receptor Associated Periodic Syndrome (TRAPS) highlights the anti- inflammatory properties of drugs for repurposing
}

Ian Todda,1, Ola H. Negma,b,g,1, Jenna Reps ${ }^{c, h}$, Paul Radforda, Grazziela Figueredoc, Elizabeth M. McDermottd, Elizabeth Drewed ${ }^{d}$, Richard J. Powella, Susan Bainbridgea, Mohamed Hameda, Sharon Crouche, Jon Garibaldic, Steve St-Gallayf, Lucy C. Fairclough ${ }^{\mathrm{a}, 2, *}$, Patrick J. Tighe $\mathrm{a}^{\mathrm{a}, 2}$

aSchool of Life Sciences, The University of Nottingham, Life Sciences Building, University Park, Nottingham, NG7 2RD, UK

${ }^{b}$ Medical Microbiology and Immunology Department, Faculty of Medicine, Mansoura University, Egypt;

'Advanced Data Analysis Centre, School of Computer Science, The University of Nottingham, Jubilee Campus, Nottingham, NG8 1BB, UK;

${ }^{d}$ Nottingham University Hospitals National Health Service Trust, Queen's Medical Centre Campus, Nottingham, NG7 2UH, UK;

eBusiness Engagement and Innovation Services, The University of Nottingham, Jubilee Campus, Nottingham, NG8 1BB, UK;

fSygnature Discovery Limited, BioCity, Pennyfoot Street, Nottingham, NG1 1GF, UK

*Corresponding author at: School of Life Sciences, University of Nottingham, Life Sciences Building, University Park, Nottingham NG7 2RD, UK.

*E-mail: lucy.fairclough@nottingham.ac.uk

Joint Authors: ${ }^{1}$ Joint first authors; ${ }^{2}$ Joint senior authors.

Present Addresses:

gSchool of Medicine, University of Nottingham, Queen's Medical Centre, Nottingham NG7 2UH, UK. hJohnson \& Johnson, Raritan, New Jersey, USA. 
Abbreviations: BCA, bicinchoninic acid; CV, coefficient of variation; MTT, 3-(4,5-dimethylthiazol2-yl)-2,5-diphenyltetrazolium bromide; PBMCs, peripheral blood mononuclear cells; RPPA, reverse phase protein array; SSMD, strictly standardised mean difference; TNFR1, tumour necrosis factor receptor-1; TRAPS, TNF receptor associated periodic syndrome; WT, wild type. 


\begin{abstract}
TNF Receptor Associated Periodic Syndrome (TRAPS) is an autoinflammatory disease caused by mutations in TNF Receptor 1 (TNFR1). Current therapies for TRAPS are limited and do not target the pro-inflammatory signalling pathways that are central to the disease mechanism. Our aim was to identify drugs for repurposing as anti-inflammatories based on their ability to down-regulate molecules associated with inflammatory signalling pathways that are activated in TRAPS. This was achieved using rigorously optimised, high through-put cell culture and reverse phase protein microarray systems to screen compounds for their effects on the TRAPS-associated inflammatory signalome. 1360 approved, publically available, pharmacologically active substances were investigated for their effects on 40 signalling molecules associated with pro-inflammatory signalling pathways that are constitutively upregulated in TRAPS. The drugs were screened at four ten-fold concentrations on cell lines expressing both wild-type (WT) TNFR1 and TRAPS-associated C33Y mutant TNFR1, or WT TNFR1 alone; signalling molecule levels were then determined in cell lysates by the reversephase protein microarray. A novel mathematical methodology was developed to rank the compounds for their ability to reduce the expression of signalling molecules in the C33Y-TNFR1 transfectants towards the level seen in the WT-TNFR1 transfectants. Seven high-ranking drugs were selected and tested by RPPA for effects on the same 40 signalling molecules in lysates of peripheral blood mononuclear cells (PBMCs) from C33Y-TRAPS patients compared to PBMCs from normal controls. The fluoroquinolone antibiotic lomefloxacin, as well as others from this class of compounds, showed the most significant effects on multiple pro-inflammatory signalling pathways that are constitutively activated in TRAPS; lomefloxacin dose-dependently significantly reduced expression of 7/40 signalling molecules across the Jak/Stat, MAPK, NF- $\kappa B$ and PI3K/AKT pathways. This study demonstrates the power of signalome screening for identifying candidates for drug repurposing.
\end{abstract}

KEYWORDS: drug repurposing, reverse-phase protein micro-array, signalome, TNF receptor associated periodic syndrome, fluoroquinolone, anti-inflammatory 


\section{Introduction}

There is great potential in the application of 'reverse-phase' protein microarray (RPPA) technology for multiplexed analysis of signalling pathways and their chemical modulation $[1,2]$. We developed a multiplexed RPPA for detecting and quantifying large numbers of intracellular signalling molecules (a 'signalome') in lysates from $\leq 20,000$ cells. Each signalling protein is detected and quantified using highly specific, verified, commercially available antibodies and a sensitive reporter system [3].

Autoinflammatory syndromes are orphan diseases with limited treatment options [4-7]. One such disease is TNF Receptor Associated Periodic Syndrome (TRAPS) caused by mutations in TNF receptor-1 (TNFR1) [8], resulting in spontaneous episodes of systemic inflammation causing morbidity and potential mortality [9-11]. Current treatment options for TRAPS include corticosteroids (with potentially major side-effects) and cytokine-blocking biologics; although the latter represent a major advance in the management of TRAPS, they are expensive, may require specialist administration, and show variable, unpredictable, limited efficacy $[12,13]$. Moreover, these anti-cytokine biologics target terminal mediators of inflammation, not central cellular processes that drive disease, which involve intracellular aggregation of misfolded mutant TNFR1 resulting in ligand-independent, constitutive activation of pro-inflammatory signalling pathways $[3,14-26]$. There would be advantages to patients and clinicians in developing drugs for TRAPS (and other autoinflammatory diseases) that are relatively cheap, taken orally, have a proven safety record and minimal side effects and target intra-cellular pro-inflammatory signalling pathways [27]. Identifying such drugs through a repurposing approach would offer major advantages in development costs and safety assessment [28-30].

We used our RPPA signalome screening method to investigate changes in proinflammatory and apoptotic signalling pathways caused by a TRAPS-associated C33Y-mutant TNFR1. Expression of the C33Y-TNFR1 in both transfected cells and TRAPS patients' peripheral blood mononuclear cells (PBMCs) caused subtle, constitutive upregulation of a wide spectrum of signalling intermediates and their phosphorylated forms associated with multiple signalling pathways, indicating a pro-inflammatory/anti-apoptotic phenotype [3]. We applied this RPPA for drug repurposing for TRAPS by screening libraries of existing, approved, off-patent, pharmacologically active compounds using a network pharmacology approach. Over 1300 
approved drugs were screened for effects on 40 signalling molecules associated with signalling pathways that we had identified as being altered in TRAPS.

\section{Materials and methods}

\subsection{Cell culture and treatment}

The human liver adenocarcinoma SK-Hep-1 endothelial-like cell line (which expresses low levels of TNFR1) was stably transfected with WT or C33Y mutant TNFRSF1A gene constructs [18]. Cells were grown in 96-well plates at a density of $\sim 1.5 \times 10^{4}$ cells per well in Dulbecco's modified Eagle's medium supplemented with 10\% fetal calf serum, 100 units $/ \mathrm{ml}$ penicillin, $10 \mathrm{mg} / \mathrm{ml}$ streptomycin, and $5 \mu \mathrm{g} / \mathrm{ml}$ blasticidin (Invitrogen Life Technologies, USA) in a $37^{\circ} \mathrm{C}$ incubator with $5 \% \mathrm{CO}_{2}$. The NINDS and Sequoia compound libraries (1360 compounds in total) were kindly supplied by MRC Technology. These compounds were dissolved in DMSO, added to the cultures at 4 ten-fold concentrations $(10 \mathrm{nM}$ to $10 \mu \mathrm{M})$, and incubated for 2 hours. (The final concentration of DMSO in the cultures was 1\%; control cultures were treated with 1\% DMSO alone.)

\subsection{Cell lysis}

The production of cell lysates and RPPA were performed for the detection of intra-cellular signaling molecules essentially as described previously [3]: the cell culture and RPPA procedures were optimized and validated at the scale required for screening hundreds of compounds. Using a liquid handling robot (BioTeck, USA), the cells were washed with ice-cold phosphate buffered saline (PBS), and lysed in 50 $\mu$ l of RIPA lysis buffer (Pierce, Thermo Fisher Scientific) and 4xSDS (0.25 M Tris $\mathrm{HCl}$ pH 6.8, 30\% Glycerol, 8\% SDS and 10\% 2-mercaptoethanol) at a ratio of 3:1, in addition to protease and phosphatase inhibitors (Pierce, Thermo Fisher Scientific) and 5 units/ml of Benzonase ${ }^{\circledR}$ Nuclease, ultrapure (Sigma Aldrich, UK). Plates were incubated on ice for 20 minutes with shaking. Finally, plates were centrifuged at $500 \mathrm{~g}$ for 5 minutes before $10 \mu \mathrm{l}$ of the supernatants were transferred into a 394 well plate using a liquid handling robot. 


\subsection{Reverse-phase protein microarray (RPPA)}

Samples were spotted in duplicate onto nitrocellulose-coated glass slides (Grace Bio-labs) with a micro-arraying robot (MicroGrid 610, Digilab, Marlborough, MA, USA) at a density of 4608 features (2304 lysates) per slide. Incubation of the slides took place overnight in blocking solution (0.2\% I-block (Tropix, Bedford, MA, USA), $0.1 \%$ Tween-20 in PBS) at $4{ }^{\circ} \mathrm{C}$ with constant rocking. After three washes of 5 minutes each, the slides were incubated with primary antibodies overnight at $4^{\circ} \mathrm{C}$ with shaking. Forty signaling molecules were detected using specific rabbit primary antibodies from Cell Signaling Technology (Table 1); these molecules are associated with

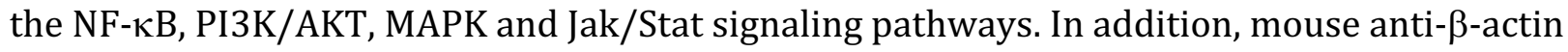
antibody (Sigma Aldrich, UK) was included as a control for protein loading. After washing, slides were incubated with infrared Licor secondary antibodies (680 CW anti-mouse Ig antibody for detection of $\beta$-actin and $800 \mathrm{CW}$ anti-rabbit Ig antibody for detection of rabbit antibodies), diluted 1:5000 in wash buffer, for 30 minutes at room temperature in the dark with shaking. Slides were washed, then dried by centrifugation at $500 \mathrm{~g}$ for 5 minutes and scanned with a Licor Odyssey scanner (LI-COR, Biosciences) at $21 \mu \mathrm{m}$ resolution at $700 \mathrm{~nm}$ (red) and $800 \mathrm{~nm}$ (green). The resultant TIFF images were processed with Genepix Pro-6 Microarray Image Analysis software (Molecular Devices Inc.) to obtain fluorescence data for each feature and generate standardized Genepix results (GPR) files. Protein signals were determined with background subtraction and normalization to the internal house-keeping targets using RPPanalyzer, a module within the R statistical language [31].

\subsection{Preparation and culture of PBMCs from TRAPS patient and control blood samples}

Blood samples were obtained following ethical approval and informed consent from five patients with the C33Y TNFR1 mutation and four healthy age and sex-matched controls. The study was approved by the Nottingham (UK) NHS Local Research Ethics Committee and by the University of Nottingham Medical School Research Ethics Committee. The experiments conformed to the principles set out in the WMA Declaration of Helsinki and the NIH Belmont report. All the patients were free from overt symptoms of inflammation at the time that blood was taken. The mean age of the patients was 49 years (range 30-71) with a male:female ratio 2:3. Three patients were being treated with anakinra and two with steroids. The four healthy age and 
sex-matched controls were of mean age 44 years, range 29-63; male:female ratio 2:2. $20 \mathrm{ml}$ blood was taken into a Falcon polypropylene tube (Becton Dickinson, NJ, USA) containing sterile citrate phosphate dextrose (ACD; Baxter Health Care, UK). Blood was then mixed 1:1 with PBS and $5 \mathrm{ml}$ Histopaque was underlayered in $50 \mathrm{ml}$ tubes followed by centrifugation at $900 \mathrm{~g}$ for 20 minutes. The PBMCs were removed, washed twice in Hank's Buffered Saline (total volume $25 \mathrm{ml}$ ). PBMCs were added to 96 well plates at $\sim 15,000$ cells/well in $200 \mu \mathrm{l} /$ well of RPMI medium and cultured with selected drugs (or 1\% DMSO alone) for 2 hours. The cells were then lysed and used in RPPA for detection of signalling molecules as described for the SK-Hep-1 cells.

\subsection{Data analysis methodology}

We developed a novel data analysis methodology utilizing mixed-effect models to identify significant effects of drugs on the signaling molecules investigated. The methodology followed two steps; the details are given in the online Supplementary Information. The first step compared the difference between the signalling pathways in WT TNFR1-transfected SK-Hep-1 cells and C33Y TNFR1-transfected SK-Hep-1 cells to identify the most interesting drugs. The aim was to find the drugs of interest that changed the signaling profile of the drug-treated C33Y-transfected cells to match that of the WT-transfected cells without drug treatment. The second step was to perform a more detailed analysis with the selected drugs of interest for effects on signaling molecule expression in PBMCs from TRAPS patients with C33Y-TNFR1 compared to PBMCs from normal healthy controls. The aim was to identify any signaling molecule where the drug had a significant interaction between the presence of the C33Y mutation and concentration of the drug on the signalling molecule's level of expression using mixed-effect models. We further investigated each significant interaction that was identified to distinguish those that significantly normalized (i.e. reduced) the expression of the signaling molecule in C33Y PBMCs towards that seen in WT PBMCs with increasing concentration of the drug, and those that exaggerated the difference in signaling molecule expression between C33Y and WT PBMCs with increasing drug concentration.

For the analysis, we used the open source statistical analysis language $\mathrm{R}$. The package lme4 was used to implement the mixed effects model and the Base statistical package was used to perform the likelihood-ratio test. The plots were produced using the ggplot2 package. 


\section{Results}

\subsection{Establishing, optimising and validating the assay methodology}

The cell culture and RPPA procedures were rigorously optimised at the scale required for screening hundreds of drugs. After optimization of robot performance, MTT and BCA assays were performed, using standard procedures, to determine SK-Hep-1 cell viability and protein recovery on robotically seeded wells, performed on separate days, to determine CVs for growth uniformity. The BCA assay intra-plate CV was $6.63 \pm 3.2 \%$ and intra-plate CV based upon MTT assays averaged $7.17 \pm 1.00 \%$ (Figure $1 \mathrm{~A}$ ).

Constitutively expressed proteins, including $\beta$-actin, $\alpha$-tubulin, Nup98, GAPDH and $\beta$ catenin were assayed by reverse-phase protein microarray from replicated 96 well culture plates, each well containing $10^{5}$ cells, to determine the uniformity of signal detection. Three 96 well plates produced on separate days were analysed, with each well printed as duplicate features on the arrays. Analysis revealed that $\beta$-actin and $\beta$-catenin showed acceptable inter-plate CVs (18.52\% for $\beta$-actin, $21.7 \%$ for $\beta$-catenin, whereas GAPDH, Nup98 and $\alpha$-tubulin gave higher CVs across all assay plates tested (31.8, $31.0 \& 23.9 \%$ respectively; see Figure $1 \mathrm{~B}) . \beta$-actin was therefore the choice for use as a normalizer, due also to the higher signal levels routinely detected in comparison to optimized anti- $\beta$-catenin antibody batches (approximately 3.5-fold higher), under standardized probing and scanning conditions.

Quality control of RPPA printing and processing was further assessed to determine print reproducibility and inter and intra-array variation. Individual samples were routinely printed across multiple arrays, and as replicates upon an array; this provided an ideal situation to monitor uniformity. Using the optimized workflow, arrays were generated and probed for $\beta$-actin. Each array contained over 760 individual features. Average inter-array actin CVs for individual samples, printed in duplicate in each array and processed as independent arrays, was $10.66 \pm 0.96 \%$ (array $n=11$ for CV calculation, from the mean of duplicate features per sample, unique sample $\mathrm{n}$ for $\mathrm{CV}$ averaging $=384$ ). Mean intra-array actin $\mathrm{CV}$, calculated from CVs of treatment replicates $=10.66 \pm 0.45 \%(n=14$ sample groups, consisting of between 32 and 64 replicates per group) (figure 1C). 


\subsection{Stimulation with PMA to examine the system capabilities for measuring signal transduction}

Verification of PMA's ability to stimulate SK-Hep-1 cells was initially assessed by measurement of HSP27 phosphorylation and thrombospondin expression in the presence of PMA, after initial testing of a range of PMA concentrations (data not shown). One hundred nM PMA was chosen as an optimal stimulus, and a 1hr exposure to PMA (figure 1D(i)), although PMA exposures up to $24 \mathrm{hrs}$ were performed (data not shown). The strictly standardized mean difference parameter (SSMD) [32, 33] $\beta$ score for pHSP27 after 1hr exposure to 100nM PMA was 6.68 , with $\beta>5$ categorized as an 'extremely strong' effect on the SSMD scale. The corresponding Z' was 0.368. Similarly, responses observed in the phosphorylation state of ERK1/2 (pERK1/2) response to the inhibitor PD98059 also give insignificant $Z^{\prime}$ values ( $\left.Z^{\prime}=0.336\right)$ despite clear visible evidence of effect (figure 1Dii), also indicated by an extremely strong SSMD $\beta=6.16$.

SSMD was therefore further examined as an alternative and possibly more robust method for QC of the initial assay, as it is designed to deal with moderate positive to negative effect differentials (e.g. RNAi experiments), and therefore has utility when observing intracellular signalling effects, where the range of response may be 1.5-4 fold maximal difference. (SSMD absolute $\beta$ scores of above 2 indicate strong thresholds for hit determination and assay QC for activation and inhibition assays where moderate effects are seen.)

Figure 1E(i-iii) demonstrates the use of the optimized screening methodology when examining 14 intracellular signaling intermediates, and the utility of the SSMD score to identify potential changes due to compound effects. As expected, PD98059 caused down-regulation of pERK1/2 and FR180204 had no effect on any signaling intermediates. SB203580 also downregulated $\mathrm{pERK} 1 / 2$ expression.

\subsection{Screening of the BioMol kinase inhibitor set}

The validation of the methodology for screening large numbers of compounds that affect signaling pathways was successfully performed by using the system to screen the 80 compound BioMol kinase inhibitor (KI) library (kindly provided by MRC Technology). Untransfected, WTTNFR1 and C33Y-TNFR1 transfected SK-Hep-1 cell lines were exposed to these KIs. The BioMol set contains a broad range of KIs, some of which inhibit kinases associated with the TRAPS disease profile. In some cases, inhibitors within the set show known, broader KI effects. This 
library therefore provided a well-defined compound group for testing the system out, with full expectation of both on and off-target positive hits. The resultant data set was analysed and correlated to both the known activities and effective concentrations of each kinase inhibitor and any detected 'promiscuous' activity or global toxicity. This represented a key test of the system's potential for larger scale application.

The 80 compounds of the BioMol inhibitor set, and DMSO control wells, were used at 3 concentrations, determined by literature analysis to give a suitable range of dilutions, namely 1 , 10 and $50 \mu \mathrm{M}$ final concentrations. All dilutions were generated in 100\% DMSO stock to preserve solubility, and final DMSO concentrations in the SK-Hep-1 cell cultures was $1 \%$, which is well tolerated by the cells, as determined by prior assays. Three independent replicate screens were performed, and all wells within the screen (each representing 15000 cells per seeding) were spotted onto nitrocellulose arrays as technical duplicates, providing 3900 features per array. Forty arrays were probed with antibodies against the target molecules shown in Table 1. Each array was concurrently probed with anti- $\beta$-actin to allow normalization for cell density differences (figure 2).

A robust version of the SSMD approach $[32,33]$ was applied to the resultant fluorescence data, after filtering spot features to remove poor spots (determined by examining spot morphology data within the results files (circularity, feature size and mean pixel intensity comparison to median pixel intensity to detect uneven feature intensity, $\%$ of pixels in feature > mean local background intensity $+2 \mathrm{SD}$ of background pixel intensity). Table 2 summarizes the key SSMD scores obtained from all the replicate experiments for those compounds which consistently gave SSMD* of $>2$ or $<-2$ across all three replicates. This is a robust measure of the hits as it is based upon whether all 3 replicates at any given treatment concentration show similar SSMDs. The data from this screen indicates that the hit rate was approximately as predicted for the BioMol set, being 5.5\% of compounds yielding hits (within the SSMD criteria stated in Table 2) with the SK-Hep-1 cells transfected with TNFR1-C33Y, and $4.2 \%$ for the TNFR1-WT SK-Hep-1 transfectant. Differential responses from the SK-Hep-1 cells expressing the TNFR1 C33Y mutation were seen, relative to cells expressing the WT receptor. For example, H-8 (nominally described as a ROCK2 inhibitor) is an isoquinaline sulphonamide derivative and one of the earliest kinase inhibitors described. It has multiple effects upon the SK-Hep-1 C33Y cell type, giving strong to extremely strong SSMD scores from 32 of 40 targets examined when 
applied at $50 \mu \mathrm{M}, 15$ of 40 targets at 10 and $1 \mu \mathrm{M}$. It is reported in the literature to lack specificity [34], and our screen indicates directly or indirectly that it affects, amongst other molecules, phosphorylation of GSK-3B, with SSMDs of $-2.47,-2.88-3.86$ at 1,10 and $50 \mu \mathrm{M}$ respectively; AKT phosphorylation (both serine and threonine sites); HSP27 phosphorylation; phosphorylation of MAPKAPK-2 (Thr334) and phosphorylation of MKK3 (Ser189)/MKK6 (Ser207). However, in the SK-Hep-1 WT transfectants, H-8 only shows SSMD* scores over 2 or below -2 for 6 target molecules, and only at the highest concentration of $\mathrm{H}-8(50 \mu \mathrm{M})$, including phospho-c-Jun (S63), phospho-Ask1(S83), PTEN, cRAF, Tak1 and Ask1. All are represented in the H-8 affected targets from the SK-Hep-1 C33Y transfected cells except for Tak1 and c-Raf, which are slightly below the thresholds stated.

Examples of more specific effects, though not entirely specific at the highest inhibitor concentrations, include: i) the anti-tumor agent Damnacathal, giving robust SSMD scores over threshold (taken as SSMD* $>2$ or $<-2$ ) for phospho-PI3K at 10 and $50 \mu \mathrm{M}$ and' at $50 \mu \mathrm{M}$, affecting phospho- ELK1, phospho-ERK1/2 and phospho-NFkB P65; ii) AG-494 is a member of the tyrphostin family of tyrosine kinase inhibitors and is a potent inhibitor of EGF receptor autophosphorylation and off-target effects are known. In our screen we saw effects of Ag-494 on phosphorylation of ELK1 (SSMD* of 2.66, $2.67 \& 3.38$ at 1, 10 and $50 \mu \mathrm{M}$ respectively) and ATF-2, with lesser effects on phospho-BAD and phospho-PI3K, but only in the SK-Hep-1 WT cells, not with the C33Y transfectant. We also saw compounds that elicit little, if any response, however the targets for many inhibitors within the BioMol set are outside the pathways linked to the targets we are examining, so this is not surprising. Off target effects are, however, still apparent, with $\mathrm{H}-8$ being a prime example.

\subsection{Identification of lead compounds from the drug repurposing library screening}

The screening of 1360 drugs in the NINDS and Sequoia compound libraries at 4 concentrations for effects on 40 signalling molecules in the 2 SK-Hep-1 cell-lines (transfected with WT or C33Y TNFR1), with duplicate lysate prints on the RPPA slides, generated 870,400 data points. An analysis method was developed to rank all the drugs at each concentration in terms of how closely the signalome of the C33Y-TNFR1 transfectants treated with the drug matched the signalome of the WT-TNFR1 transfectants without the drug (see the online Appendix A - Supplementary Information). Thus, the highest-ranking drugs gave the best 
alignment of the C33Y signalome with the untreated WT signalome. This generated $>5400$ ranks with lower scores corresponding to higher ranks. The range of scores was 11 (ranked 1) to >3.8 $x 10^{6}$. Figure 3a shows the cumulative profile of scores (expressed as binned $\log _{10}$ values) for all drugs at all concentrations; this shows a reasonably Gaussian distribution with geometric mean score 102 (2.01 log units) and standard deviation 0.26 log units.

The drug/concentration combinations ranked 1-35, with scores 11-31 (Table 3) were examined to select a small number for further investigation (see the Discussion for a broader consideration of the top-ranked drugs). The selection criteria were that drugs should have proven safety profiles in humans, they should represent a range of drug classes in terms of established therapeutic uses, and that there should be literature-based evidence of them showing anti-inflammatory effects. The drugs were also ranked on the median score of all four of the drug concentrations tested. On this basis, the drugs selected (with highest/median score) were: cefamandole nafate (14/38) - a cephalosporin antibiotic; lomefloxacin hydrochloride (19/55) - a fluoroquinolone antibiotic; estradiol-3-sulfate (19/122) - a female steroid hormone; ketoprofen (22/48) - a non-steroidal anti-inflammatory drug; fosfomycin (22/65) - a phosphonic acid antibiotic. In addition, methylprednisolone (30/54) was chosen as a positive control because its primary use is as an anti-inflammatory/immunosuppressive drug; fluoxetine (471/598) - a selective serotonin reuptake inhibitor with no known anti-inflammatory effects - was chosen as a negative control.

Further validation of this choice of compounds is provided by the presentation of data in figure $3 \mathrm{~b}$, which shows all the compounds tested, but clustered into structural families and positioned according to the mean score logged to base 10 of each cluster and the number of compounds in the cluster. This highlights the fluoroquinolones (and closely related quinolones), the estradiols and cephalosporins, together with the 'positive control' glucocorticoids. Berberines were also highlighted in this analysis of the data (figure $3 \mathrm{~b}$ ), but the highest ranking of these, ethaverine, was ranked only $114^{\text {th }}(100 \mathrm{~nm}$, score 43$)$. In addition, a Bayesian model was developed from the tested compounds and their scores to classify and predict active and inactive compounds based on structural features. This model was applied to all untested compounds listed in ChEMBL as being in phase IV clinical trials with molecular weights between 100 and 600. Figure 3c shows that this, again, highlighted quinolones, cephalosporins, estradiols and glucocorticoids (and other steroids) as the main compounds predicted to be active in our 
signalome assay, thereby further supporting our choice of compounds for further testing. (Compounds shown as inverted triangles fail 'Lipinski's rule of five' and are unlikely to function as orally active drugs in humans [35].)

\subsection{Further screening of lead compounds on peripheral blood mononuclear cells from TRAPS patients and healthy controls}

The seven selected drugs indicated above were screened at 4 ten-fold concentrations on unstimulated, cultured PBMCs from 5 C33Y-TNFR1 TRAPS patients and 4 normal healthy controls. Expression of the same 40 signalling molecules detected in the SK-Hep-1 transfectants was analysed by protein microarray in lysates made from the PBMCs after 2 hours of treatment with the drugs. The resulting data was analysed using mixed-effect models to identify drugs that induced a significant dose-dependent reduction of signalling molecules in the TRAPS patients' PBMCs towards the level seen in control PBMCs, or caused a dose-dependent increase in signalling molecule expression in TRAPS patients' PBMCs relative to levels in control PBMCs (for mathematical details, see the online Supplementary Information). A significance level of $10 \%$ ( $p$ $\leq 0.1$ ) was employed due to the relatively small sample size. The results for signalling molecules whose expression in TRAPS patients' PBMCs were significantly, dose-dependently, decreased towards normal levels, or increased away from normal levels, by particular drugs are summarised in table 4. Cefamandole nafate and ketoprofen each reduced expression of only a single signalling molecule in TRAPS patients' PBMCs (PI3K.P110 and SOCS3, respectively, with the latter being an inhibitor of cytokine signalling), and both of these drugs upregulated expression of numerous signalling molecules (12 and 7, respectively). Only upregulation of signalling molecules in TRAPS patient's PBMCs was observed with estradiol-3-sulphate, fosfomycin, and even with methylprednisolone; no significant effects were observed with fluoxetine. By contrast, lomefloxacin showed significant dose-dependent reduction of seven signalling molecules in TRAPS patients' PBMCs across all four different pro-inflammatory signalling pathways, and upregulation of four signalling molecules (table 4). Thus, this identified lomefloxacin as a lead compound in terms of down-regulating the pro-inflammatory signalome of TRAPS patients' PBMCs.

Lomefloxacin is a member of the fluoroquinolone/quinolone class of antibiotics that act as 
bacterial DNA gyrase inhibitors. Indeed, it is of note that the highest-ranking compound in the initial screen of 1340 drugs was the quinolone antibiotic piromidic acid (score 11) and that the fluoroquinolone ofloxacin was ranked twentieth (lomefloxacin was ranked ninth). Furthermore, piromidic acid, lomefloxacin and ofloxacin were amongst only 31 out of the 1360 drugs for whom all four drug concentrations gave scores $\leq 100$ (three of the others were the recognised antiinflammatory drugs prednisolone, methylprednisolone and hydrocortisone). Also of relevance is the empirical observation, made independently of the current findings, that a TRAPS patient with the C33Y-TNFR1 mutation, who was given the fluoroquinolone moxifloxacin to treat a pneumococcal chest infection also showed dramatic improvements in her TRAPS symptoms, and this was maintained for two years whilst moxifloxacin administration continued [36]. However, it is possible that this therapeutic effect was due to the drug's antimicrobial activity; furthermore, administration of fluoroquinolones to three other C33Y-TNFR1 TRAPS patients showed no benefit [36]. Moxifloxacin was the third highest ranked fluoroquinolone in the library screen (score 50) after lomefloxacin and ofloxacin. This adds further weight to the potential antiinflammatory effects of fluoroquinolones in TRAPS. Moxifloxacin was not screened for effects on signaling molecules in TRAPS patients' PBMCs in the present study as it fell outside of the top 35 ranked compounds; however, it remains of interest to test moxifloxacin in this assay.

\section{Discussion}

We previously demonstrated that multiple pro-inflammatory signaling pathways are activated in TRAPS [3]. Current therapies include corticosteroids with significant side-effects when used long-term, and expensive biologics that neutralize cytokines produced as a consequence of the disease mechanisms, rather than targeting the underlying aetiology $[12,27]$. To address this, we developed, optimized and validated a protein microarray-based network pharmacology approach to identify repurposing drugs that simultaneously target multiple signaling pathways that are activated in TRAPS. Repurposing of existing drugs is a cost-effective and efficient means of exploiting drugs whose safety and pharmacological profiles are established, reducing development time and cost for new therapeutic indications. Development costs of repurposed drugs are about $60 \%$ that of novel compounds, and they are three times more likely to gain market approval [37]. 
The first stage of the screening strategy utilizing the SK-Hep-1 C33Y and WT TNFR1 transfectants as the target cells highlighted several compounds of interest within the top ranks (Table 3). Three quinolone/fluoroquinolone antibiotics are represented in the top 35 compounds: piromidic acid (ranked 1 and 22), lomefloxacin (ranked 8) and ofloxacin (ranked 19). Piromidic acid is a first-generation quinolone antibiotic that is no longer widely used, which is why it was not chosen for further analysis in this study; lomefloxacin and ofloxacin are second generation fluoroquinolone antibiotics. Significant evidence of in vivo and in vitro antiinflammatory activities of fluoroquinolones has been reported previously [38-42]. In particular, with regard to the effects of fluoroquinolones on pro-inflammatory signaling intermediates, it has been reported that norfloxacin and gemifloxacin reduce NFKB expression and activation [43-45], and that garenoxacin inhibits Erk1/2 phosphorylation [46]. Moxifloxacin also inhibits Erk1/2, JNK and NFKB [47-49]. Certain derivatives of fluoroquinolones have been reported selectively to inhibit PI3K $\gamma$ [50]. Four other antibiotics occur in the top 35 compounds: these are the cephalosporins cefamandole nafate (ranked 2 and 13) and cefuroxime sodium (ranked 3); metampicillin sodium (ranked 9) and fosfomycin (ranked 12). There is some evidence for immune-modulatory properties of cephalosporins [51]. Fosfomycin has been reported to modulate cytokine production by inhibiting NFKB activation [52].

Four cyclo-oxygenase (COX) inhibitors are represented in the top 35 compounds: carprofen (ranked 4 and 28), ketoprofen (ranked 14), tolfenamic acid (ranked 20) and mefenamic acid (ranked 23). Carprofen is designated for veterinary use only. With respect to ketoprofen, although it was observed only to down-regulate the inhibitor of cytokine signalling SOCS3 in the current study, others have reported that it suppresses production of IL-1 $\beta$ and TNF $\alpha$ in LPS-stimulated human dental pulp cells, and to inhibit phosphorylation of ERK and JNK [53].

Methylprednisolone, a widely used anti-inflammatory and immunosuppressive corticosteroid drug, is ranked 32. Hecogenin (ranked 29) is a steroidal saponin derived from plants (genus Agave - 'sisal') used pharmaceutically as a precursor for steroid hormone synthesis; it is reported to inhibit the synthesis of pro-inflammatory cytokines like IL-1 $\beta$ [54]. Particularly interesting is the occurrence of several oestrogens amongst the top 35 compounds: estriol benzyl ether (ranked 6 and 10), estrdiol-3-sulphate (ranked 7) and estradiol acetate (ranked 15). It is well recognised that certain autoimmune diseases (e.g. multiple sclerosis and 
rheumatoid arthritis) ameliorate during pregnancy in female patients, when levels of estriol increase. A recently published study provides some evidence that female patients with relapsing/remitting multiple sclerosis who were treated with estriol in combination with glatiramer acetate experienced fewer relapses of disease than patients receiving glatiramer acetate alone [55].

Three vitamins are represented in the top 35 compounds, namely: retinol/vitamin A (ranked 17); lapachol (ranked 34), which is a derivative of vitamin K; and ergocalciferol/vitamin $\mathrm{D}_{2}$ (ranked 35). There is good evidence for immunomodulatory activity of vitamin $\mathrm{D}$, and association between poor vitamin D status and increased risk of various autoimmune diseases [56]; evidence also indicates that vitamin D may reduce the risk of severe exacerbations of asthma [57].

Amlexanox (ranked 30) is an anti-inflammatory agent used for the treatment of aphthous ulcers and (in Japan) it is used to treat bronchial asthma, allergic rhinitis and conjunctivitis. It

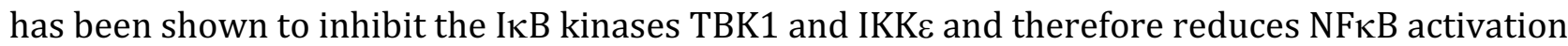
[58-61]. There are also reports of amlexanox inhibiting G protein-coupled receptor kinase 5 [62, $63]$ and that it binds to the S100 proteins A4 and A13 [64,65]. This may also help to explain the anti-inflammatory effects of amlexanox as S100A4 is reportedly a TLR4 agonist [66].

Fenspiride hydrochloride (ranked 25) is a bronchodilator with anti-inflammatory properties that may act as an antagonist to $\alpha$-adrenergic and $\mathrm{H} 1$ histamine receptors. Pindolol (ranked 18) and ephedrine hydrochloride (ranked 26) are $\beta$-adrenoceptor ligands: they are an antagonist and agonist, respectively, but with some structural similarities.

The second stage screening of selected compounds on PBMCs from TRAPS patients and healthy controls highlighted the anti-inflammatory effects of the fluoroquinolone antibiotic lomefloxacin, as this down-regulated signaling molecules associated with four pro-inflammatory signaling pathways that are activated in TRAPS. Independent of this, the report that a TRAPS patient showed significant therapeutic anti-inflammatory benefit from treatment with the fluoroquinolone moxifloxacin [36] adds significant further support to the potential of fluoroquinolones as anti-inflammatory agents in the management of TRAPS. Targeting signaling molecules in inflammatory diseases is exemplified by the recent approval of JAK inhibitors in rheumatoid arthritis [67]. 
Figure 4 summarises the effects of lomefloxacin that we observed on signaling pathways in TRAPS patients' PBMCs: the expression of molecules shown in red was dose-dependently down-regulated by lomefloxacin, those in green were upregulated, and those in blue were unaffected. This is, necessarily, a simplified representation as many signalling molecules associate with more than one signalling pathway, and can have opposing effects in different contexts. However, figure 4 demonstrates how lomefloxacin mediates anti-inflammatory effects by down-regulating pro-inflammatory signalling molecules (p38MAPK, cSrc, RIP, AKT1/2, Jak2) and upregulating anti-inflammatory molecules (e.g. cIAP1/2) - even the cRaf-Erk1/2 pathway can have anti-inflammatory actions by inhibiting Stat3 [68]. Conversely, the down-regulation of the PI3K inhibitor PTEN may be a feedback consequence of the negative regulation of this pathway by the down-regulation of phospho-AKT1/2 by lomefloxacin.

The present study does not elucidate the molecular mechanisms of the effects of lomefloxacin on pro-inflammatory signalling pathways, which will be the subject of further investigations. Certain fluoroquinolone derivatives have been reported to selectively inhibit PI3K $\gamma$ [50]; however, lomefloxacin appears to have broader effects on a number of proinflammatory signalling pathways. The findings reported here do, however, provide a mechanistic basis for the independent, empirical observation of the anti-inflammatory therapeutic benefit of moxifloxacin in a TRAPS patient [36], and concurs with other reports of inhibitory effects of moxifloxacin on MAP-kinases and NFKB [47-49]. However, it cannot be excluded that the beneficial effects of moxifloxacin in this patient were due to its anti-microbial activity against infections that might otherwise have triggered TRAPS-associated inflammation; furthermore, moxifloxacin and ciprofloxacin were not beneficial in three other TRAPS patients with the C33Y-TNFR1 mutation [36].

When considering broadening the therapeutic use of FQs beyond use as anti-microbials, it is important to bear in mind that these drugs can have significant toxic side effects and their application is restricted in certain groups, particularly children [69, 70]; also, the dangers of promoting microbial resistance through wider use must be considered [71]. Thus, generating modified derivatives, or finding structurally-related alternatives to FQs that have reduced toxicity and anti-microbial activity but show enhanced anti-inflammatory activity, could provide a valuable source of novel anti-inflammatory agents. More generally, the present study demonstrates the value cell-lysate RPPA for screening large numbers of chemicals for effects on 
multiple signaling molecules in the context of drug development and repurposing.

\section{Funding information}

This work was supported by grants from the Developmental Pathway Funding Scheme (ref. MRJ0051501) and the Proximity to Discovery Funding Scheme (ref. P2D15002) of the Medical Research Council UK; it was also supported by funding from The Jones 1986 Charitable Trust.

\section{Notes}

The authors declare no competing financial interest.

\section{Acknowledgments}

We are grateful to MRC Technology for providing the NINDS and Sequoia compound libraries.

We are grateful to all the volunteer subjects who contributed clinical and control samples for this project.

\section{References}

[1] M. Sevecka, G. MacBeath, State-based discovery: a multidimensional screen for smallmolecule modulators of EGF signaling, Nature methods 3(10) (2006) 825-31.

[2] M. Sevecka, A. Wolf-Yadlin, G. MacBeath, Lysate microarrays enable high-throughput, quantitative investigations of cellular signaling, Molecular \& cellular proteomics : MCP 10(4) (2011) M110 005363.

[3] O.H. Negm, H.A. Mannsperger, E.M. McDermott, E. Drewe, R.J. Powell, I. Todd, L.C. Fairclough, P.J. Tighe, A pro-inflammatory signalome is constitutively activated by C33Y mutant TNF receptor 1 in TNF receptor-associated periodic syndrome (TRAPS), Eur J Immunol 44(7) (2014) 2096-110.

[4] A.A. de Jesus, S.W. Canna, Y. Liu, R. Goldbach-Mansky, Molecular mechanisms in genetically defined autoinflammatory diseases: disorders of amplified danger signaling, Annu Rev Immunol 33 (2015) 823-74.

[5] S. Pathak, M.F. McDermott, S. Savic, Autoinflammatory diseases: update on classification diagnosis and management, Journal of clinical pathology 70(1) (2017) 1-8.

[6] D. Peckham, T. Scambler, S. Savic, M.F. McDermott, The burgeoning field of innate immunemediated disease and autoinflammation, The Journal of pathology 241(2) (2017) 123-139. [7] S.R. Harrison, D. McGonagle, S. Nizam, S. Jarrett, J. van der Hilst, M.F. McDermott, S. Savic, Anakinra as a diagnostic challenge and treatment option for systemic autoinflammatory disorders of undefined etiology, JCI Insight 1(6) (2016) e86336.

[8] M.F. McDermott, I. Aksentijevich, J. Galon, E.M. McDermott, B.W. Ogunkolade, M. Centola, E. Mansfield, M. Gadina, L. Karenko, T. Pettersson, J. McCarthy, D.M. Frucht, M. Aringer, Y. Torosyan, A.M. Teppo, M. Wilson, H.M. Karaarslan, Y. Wan, I. Todd, G. Wood, R. Schlimgen, T.R. Kumarajeewa, S.M. Cooper, J.P. Vella, C.I. Amos, J. Mulley, K.A. Quane, M.G. Molloy, A. Ranki, R.J. Powell, G.A. Hitman, J.J. O'Shea, D.L. Kastner, Germline mutations in the extracellular domains of the $55 \mathrm{kDa}$ 
TNF receptor, TNFR1, define a family of dominantly inherited autoinflammatory syndromes, Cell 97(1) (1999) 133-44.

[9] S. Federici, M.P. Sormani, S. Ozen, H.J. Lachmann, G. Amaryan, P. Woo, I. Kone-Paut, N. Dewarrat, L. Cantarini, A. Insalaco, Y. Uziel, D. Rigante, P. Quartier, E. Demirkaya, T. Herlin, A. Meini, G. Fabio, T. Kallinich, S. Martino, A.Y. Butbul, A. Olivieri, J. Kuemmerle-Deschner, B. Neven, A. Simon, H. Ozdogan, I. Touitou, J. Frenkel, M. Hofer, A. Martini, N. Ruperto, M. Gattorno, O. Paediatric Rheumatology International Trials, P. Eurofever, Evidence-based provisional clinical classification criteria for autoinflammatory periodic fevers, Ann Rheum Dis 74(5) (2015) 799805.

[10] H.J. Lachmann, R. Papa, K. Gerhold, L. Obici, I. Touitou, L. Cantarini, J. Frenkel, J. Anton, I. Kone-Paut, M. Cattalini, B. Bader-Meunier, A. Insalaco, V. Hentgen, R. Merino, C. Modesto, N. Toplak, R. Berendes, S. Ozen, R. Cimaz, A. Jansson, P.A. Brogan, P.N. Hawkins, N. Ruperto, A. Martini, P. Woo, M. Gattorno, t.E. Paediatric Rheumatology International Trials Organisation, P. the Eurofever, The phenotype of TNF receptor-associated autoinflammatory syndrome (TRAPS) at presentation: a series of 158 cases from the Eurofever/EUROTRAPS international registry, Ann Rheum Dis 73(12) (2014) 2160-7.

[11] M. Piram, I. Kone-Paut, H.J. Lachmann, J. Frenkel, S. Ozen, J. Kuemmerle-Deschner, S. Stojanov, A. Simon, M. Finetti, M.P. Sormani, A. Martini, M. Gattorno, N. Ruperto, E. Eurofever, n. the Paediatric Rheumatology International Trials Organisation, Validation of the auto-inflammatory diseases activity index (AIDAI) for hereditary recurrent fever syndromes, Ann Rheum Dis 73(12) (2014) 2168-73.

[12] N.M. ter Haar, M. Oswald, J. Jeyaratnam, J. Anton, K.S. Barron, P.A. Brogan, L. Cantarini, C. Galeotti, G. Grateau, V. Hentgen, M. Hofer, T. Kallinich, I. Kone-Paut, H.J. Lachmann, H. Ozdogan, S. Ozen, R. Russo, A. Simon, Y. Uziel, C. Wouters, B.M. Feldman, S.J. Vastert, N.M. Wulffraat, S.M. Benseler, J. Frenkel, M. Gattorno, J.B. Kuemmerle-Deschner, Recommendations for the management of autoinflammatory diseases, Ann Rheum Dis 74(9) (2015) 1636-44.

[13] P.M. Vaitla, P.M. Radford, P.J. Tighe, R.J. Powell, E.M. McDermott, I. Todd, E. Drewe, Role of interleukin-6 in a patient with tumor necrosis factor receptor-associated periodic syndrome: assessment of outcomes following treatment with the anti-interleukin- 6 receptor monoclonal antibody tocilizumab, Arthritis and rheumatism 63(4) (2011) 1151-5.

[14] I. Todd, P.M. Radford, K.A. Draper-Morgan, R. McIntosh, S. Bainbridge, P. Dickinson, L. Jamhawi, M. Sansaridis, M.L. Huggins, P.J. Tighe, R.J. Powell, Mutant forms of tumour necrosis factor receptor I that occur in TNF-receptor-associated periodic syndrome retain signalling functions but show abnormal behaviour, Immunology 113(1) (2004) 65-79.

[15] M.L. Huggins, P.M. Radford, R.S. McIntosh, S.E. Bainbridge, P. Dickinson, K.A. Draper-Morgan, P.J. Tighe, R.J. Powell, I. Todd, Shedding of mutant tumor necrosis factor receptor superfamily $1 \mathrm{~A}$ associated with tumor necrosis factor receptor-associated periodic syndrome: differences between cell types, Arthritis Rheum 50(8) (2004) 2651-9.

[16] N. Yousaf, D.J. Gould, E. Aganna, L. Hammond, R.M. Mirakian, M.D. Turner, G.A. Hitman, M.F. McDermott, Y. Chernajovsky, Tumor necrosis factor receptor I from patients with tumor necrosis factor receptor-associated periodic syndrome interacts with wild-type tumor necrosis factor receptor I and induces ligand-independent NF-kappaB activation, Arthritis and rheumatism 52(9) (2005) 2906-16.

[17] A.A. Lobito, F.C. Kimberley, J.R. Muppidi, H. Komarow, A.J. Jackson, K.M. Hull, D.L. Kastner, G.R. Screaton, R.M. Siegel, Abnormal disulfide-linked oligomerization results in ER retention and altered signaling by TNFR1 mutants in TNFR1-associated periodic fever syndrome (TRAPS), Blood 108(4) (2006) 1320-7. 
[18] S.L. Rebelo, S.E. Bainbridge, M.R. Amel-Kashipaz, P.M. Radford, R.J. Powell, I. Todd, P.J. Tighe, Modeling of tumor necrosis factor receptor superfamily $1 \mathrm{~A}$ mutants associated with tumor necrosis factor receptor-associated periodic syndrome indicates misfolding consistent with abnormal function, Arthritis Rheum 54(8) (2006) 2674-87.

[19] I. Todd, P.M. Radford, N. Daffa, S.E. Bainbridge, R.J. Powell, P.J. Tighe, Mutant tumor necrosis factor receptor associated with tumor necrosis factor receptor-associated periodic syndrome is altered antigenically and is retained within patients' leukocytes, Arthritis Rheum 56(8) (2007) 2765-73.

[20] S.L. Rebelo, M.R. Amel-Kashipaz, P.M. Radford, S.E. Bainbridge, R. Fiets, J. Fang, E.M. McDermott, R.J. Powell, I. Todd, P.J. Tighe, Novel markers of inflammation identified in tumor necrosis factor receptor-associated periodic syndrome (TRAPS) by transcriptomic analysis of effects of TRAPS-associated tumor necrosis factor receptor type I mutations in an endothelial cell line, Arthritis Rheum 60(1) (2009) 269-80.

[21] A. Simon, H. Park, R. Maddipati, A.A. Lobito, A.C. Bulua, A.J. Jackson, J.J. Chae, R. Ettinger, H.D. de Koning, A.C. Cruz, D.L. Kastner, H. Komarow, R.M. Siegel, Concerted action of wild-type and mutant TNF receptors enhances inflammation in TNF receptor 1-associated periodic fever syndrome, Proc Natl Acad Sci U S A 107(21) (2010) 9801-6.

[22] A.C. Bulua, A. Simon, R. Maddipati, M. Pelletier, H. Park, K.Y. Kim, M.N. Sack, D.L. Kastner, R.M. Siegel, Mitochondrial reactive oxygen species promote production of proinflammatory cytokines and are elevated in TNFR1-associated periodic syndrome (TRAPS), J Exp Med 208(3) (2011) 51933.

[23] L.J. Dickie, A.M. Aziz, S. Savic, O.M. Lucherini, L. Cantarini, J. Geiler, C.H. Wong, R. Coughlan, T. Lane, H.J. Lachmann, P.N. Hawkins, P.A. Robinson, P. Emery, D. McGonagle, M.F. McDermott, Involvement of $\mathrm{X}$-box binding protein 1 and reactive oxygen species pathways in the pathogenesis of tumour necrosis factor receptor-associated periodic syndrome, Ann Rheum Dis 71(12) (2012) 2035-43.

[24] E. Greco, A. Aita, P. Galozzi, A. Gava, P. Sfriso, O.H. Negm, P. Tighe, F. Caso, F. Navaglia, E. Dazzo, M. De Bortoli, A. Rampazzo, L. Obici, S. Donadei, G. Merlini, M. Plebani, I. Todd, D. Basso, L. Punzi, The novel S59P mutation in the TNFRSF1A gene identified in an adult onset TNF receptor associated periodic syndrome (TRAPS) constitutively activates NF-kappaB pathway, Arthritis research \& therapy 17 (2015) 93.

[25] L.C. Fairclough, A.A. Stoop, O.H. Negm, P.M. Radford, P.J. Tighe, I. Todd, Tumour necrosis factor receptor I blockade shows that TNF-dependent and TNF-independent mechanisms synergise in TNF receptor associated periodic syndrome, Eur J Immunol 45(10) (2015) 2937-44. [26] A.F. Agyemang, S.R. Harrison, R.M. Siegel, M.F. McDermott, Protein misfolding and dysregulated protein homeostasis in autoinflammatory diseases and beyond, Semin Immunopathol 37(4) (2015) 335-47.

[27] A. Marcuzzi, E. Piscianz, E. Valencic, L. Monasta, L. Vecchi Brumatti, A. Tommasini, To Extinguish the Fire from Outside the Cell or to Shutdown the Gas Valve Inside? Novel Trends in Anti-Inflammatory Therapies, International journal of molecular sciences 16(9) (2015) 21277-93. [28] Y. Cha, T. Erez, I.J. Reynolds, D. Kumar, J. Ross, G. Koytiger, R. Kusko, B. Zeskind, S. Risso, E. Kagan, S. Papapetropoulos, I. Grossman, D. Laifenfeld, Drug repurposing from the perspective of pharmaceutical companies, British journal of pharmacology (2017).

[29] D. Sardana, C. Zhu, M. Zhang, R.C. Gudivada, L. Yang, A.G. Jegga, Drug repositioning for orphan diseases, Briefings in bioinformatics 12(4) (2011) 346-56.

[30] K. Shameer, B. Readhead, J.T. Dudley, Computational and experimental advances in drug repositioning for accelerated therapeutic stratification, Current topics in medicinal chemistry 15(1) (2015) 5-20. 
[31] H.A. Mannsperger, S. Gade, F. Henjes, T. Beissbarth, U. Korf, RPPanalyzer: Analysis of reverse-phase protein array data, Bioinformatics 26(17) (2010) 2202-3.

[32] X.D. Zhang, A pair of new statistical parameters for quality control in RNA interference highthroughput screening assays, Genomics 89(4) (2007) 552-61.

[33] X.D. Zhang, Illustration of SSMD, z score, SSMD*, $z^{*}$ score, and t statistic for hit selection in RNAi high-throughput screens, Journal of biomolecular screening 16(7) (2011) 775-85.

[34] J. Bain, L. Plater, M. Elliott, N. Shpiro, C.J. Hastie, H. McLauchlan, I. Klevernic, J.S. Arthur, D.R. Alessi, P. Cohen, The selectivity of protein kinase inhibitors: a further update, The Biochemical journal 408(3) (2007) 297-315.

[35] C.A. Lipinski, F. Lombardo, B.W. Dominy, P.J. Feeney, Experimental and computational approaches to estimate solubility and permeability in drug discovery and development settings, Adv Drug Deliv Rev 46(1-3) (2001) 3-26.

[36] E. Drewe, R.J. Powell, E.M. McDermott, Comment on: Failure of anti-TNF therapy in TNF receptor 1-associated periodic syndrome (TRAPS), Rheumatology 46(12) (2007) 1865-6.

[37] S.H.J. Beachy, S.G.; Olson, S.; Berger, A.C. , Drug Repurposing and Repositioning; Workshop Summary (2014). The National Academies Press, Washington, D.C., 2014.

[38] C. Beisswenger, A. Honecker, A. Kamyschnikow, M. Bischoff, T. Tschernig, R. Bals, Moxifloxacin modulates inflammation during murine pneumonia, Respir Res 15 (2014) 82.

[39] R. Fukumoto, L.H. Cary, N.V. Gorbunov, E.D. Lombardini, T.B. Elliott, J.G. Kiang, Ciprofloxacin modulates cytokine/chemokine profile in serum, improves bone marrow repopulation, and limits apoptosis and autophagy in ileum after whole body ionizing irradiation combined with skinwound trauma, PLoS One 8(3) (2013) e58389.

[40] H. Ogino, M. Fujii, M. Ono, K. Maezawa, S. Hori, J. Kizu, In vivo and in vitro effects of fluoroquinolones on lipopolysaccharide-induced pro-inflammatory cytokine production, Journal of infection and chemotherapy : official journal of the Japan Society of Chemotherapy 15(3) (2009) 168-73.

[41] F. Sachse, C. von Eiff, K. Becker, C. Rudack, Anti-inflammatory effects of ciprofloxacin in S. aureus Newman induced nasal inflammation in vitro, Journal of inflammation 5 (2008) 11.

[42] R. Tsivkovskii, M. Sabet, Z. Tarazi, D.C. Griffith, O. Lomovskaya, M.N. Dudley, Levofloxacin reduces inflammatory cytokine levels in human bronchial epithelia cells: implications for aerosol MP-376 (levofloxacin solution for inhalation) treatment of chronic pulmonary infections, FEMS immunology and medical microbiology 61(2) (2011) 141-6.

[43] I. Gomez-Hurtado, P. Zapater, P. Bellot, S. Pascual, M. Perez-Mateo, J. Such, R. Frances, Interleukin-10-mediated heme oxygenase 1-induced underlying mechanism in inflammatory down-regulation by norfloxacin in cirrhosis, Hepatology 53(3) (2011) 935-44.

[44] J.Y. Kan, Y.L. Hsu, Y.H. Chen, T.C. Chen, J.Y. Wang, P.L. Kuo, Gemifloxacin, a fluoroquinolone antimicrobial drug, inhibits migration and invasion of human colon cancer cells, BioMed research international 2013 (2013) 159786.

[45] P. Zapater, R. Cano, L. Llanos, A.J. Ruiz-Alcaraz, S. Pascual, C. Barquero, R. Moreu, P. Bellot, J.F. Horga, C. Munoz, J. Perez, P. Garcia-Penarrubia, M. Perez-Mateo, J. Such, R. Frances, Norfloxacin modulates the inflammatory response and directly affects neutrophils in patients with decompensated cirrhosis, Gastroenterology 137(5) (2009) 1669-79 e1.

[46] S. Hara, Y. Ishimatsu, H. Mukae, N. Sakamoto, T. Kakugawa, H. Fujita, A. Hara, S. Kohno, Antiinflammatory effects of garenoxacin on IL-8 production and ERK1/2 activation induced by lipopolysaccharides in A549 and THP-1 cells, European journal of pharmacology 668(1-2) (2011) 264-70.

[47] H. Blau, K. Klein, I. Shalit, D. Halperin, I. Fabian, Moxifloxacin but not ciprofloxacin or azithromycin selectively inhibits IL-8, IL-6, ERK1/2, JNK, and NF-kappaB activation in a cystic 
fibrosis epithelial cell line, American journal of physiology. Lung cellular and molecular physiology 292(1) (2007) L343-52.

[48] I. Shalit, D. Halperin, D. Haite, A. Levitov, J. Romano, N. Osherov, I. Fabian, Anti-inflammatory effects of moxifloxacin on IL-8, IL-1beta and TNF-alpha secretion and NFkappaB and MAP-kinase activation in human monocytes stimulated with Aspergillus fumigatus, The Journal of antimicrobial chemotherapy 57(2) (2006) 230-5.

[49] S. Werber, I. Shalit, I. Fabian, G. Steuer, T. Weiss, H. Blau, Moxifloxacin inhibits cytokineinduced MAP kinase and NF-kappaB activation as well as nitric oxide synthesis in a human respiratory epithelial cell line, The Journal of antimicrobial chemotherapy 55(3) (2005) 293-300. [50] S. Sha, Han, H-W., Gao, F., Liu, T-B., Li, Z., Xu, C., Zhong, W-Q. and Zhu, H-L., Discovery of fluoroquinolone derivatives as potent, selective inhibitors of PI3Kgamma, Med Chem Commun 6 (2015) 2029-2035.

[51] S.C. Tauber, R. Nau, Immunomodulatory properties of antibiotics, Curr Mol Pharmacol 1(1) (2008) 68-79.

[52] Y. Yoneshima, T. Ichiyama, H. Ayukawa, T. Matsubara, S. Furukawa, Fosfomycin inhibits NFkappaB activation in U-937 and Jurkat cells, International journal of antimicrobial agents 21(6) (2003) 589-92.

[53] E.K. Choi, S.H. Kim, I.C. Kang, J.Y. Jeong, J.T. Koh, B.N. Lee, W.M. Oh, K.S. Min, J.E. Nor, Y.C. Hwang, Ketoprofen inhibits expression of inflammatory mediators in human dental pulp cells, Journal of endodontics 39(6) (2013) 764-7.

[54] J.S. Quintans, R.S. Barreto, W. de Lucca, Jr., C.F. Villarreal, C.M. Kaneto, M.B. Soares, A. Branco, J.R. Almeida, A.G. Taranto, A.R. Antoniolli, R.M. Freitas, L.J. Quintans, Jr., Evidence for the involvement of spinal cord-inhibitory and cytokines-modulatory mechanisms in the antihyperalgesic effect of hecogenin acetate, a steroidal sapogenin-acetylated, in mice, Molecules 19(6) (2014) 8303-16.

[55] R.R. Voskuhl, H. Wang, T.C. Wu, N.L. Sicotte, K. Nakamura, F. Kurth, N. Itoh, J. Bardens, J.T. Bernard, J.R. Corboy, A.H. Cross, S. Dhib-Jalbut, C.C. Ford, E.M. Frohman, B. Giesser, D. Jacobs, L.H. Kasper, S. Lynch, G. Parry, M.K. Racke, A.T. Reder, J. Rose, D.M. Wingerchuk, A.J. MacKenzieGraham, D.L. Arnold, C.H. Tseng, R. Elashoff, Estriol combined with glatiramer acetate for women with relapsing-remitting multiple sclerosis: a randomised, placebo-controlled, phase 2 trial, The Lancet. Neurology 15(1) (2016) 35-46.

[56] N. Agmon-Levin, E. Theodor, R.M. Segal, Y. Shoenfeld, Vitamin D in systemic and organspecific autoimmune diseases, Clin Rev Allergy Immunol 45(2) (2013) 256-66.

[57] C.C.J. Martineau A.R., Urashima M., Jensen M., Griffiths A.P., Nurmatov U., Sheikh A., Griffiths C.J., Vitamin D for the management of asthma, Cochrane Database of Systematic Reviews (9) (2016).

[58] S.M. Reilly, S.H. Chiang, S.J. Decker, L. Chang, M. Uhm, M.J. Larsen, J.R. Rubin, J. Mowers, N.M. White, I. Hochberg, M. Downes, R.T. Yu, C. Liddle, R.M. Evans, D. Oh, P. Li, J.M. Olefsky, A.R. Saltiel, An inhibitor of the protein kinases TBK1 and IKK-varepsilon improves obesity-related metabolic dysfunctions in mice, Nat Med 19(3) (2013) 313-21.

[59] S. Challa, J.P. Guo, X. Ding, C.X. Xu, Y. Li, D. Kim, M.A. Smith, D.W. Cress, D. Coppola, E.B. Haura, J.Q. Cheng, IKBKE Is a Substrate of EGFR and a Therapeutic Target in Non-Small Cell Lung Cancer with Activating Mutations of EGFR, Cancer research 76(15) (2016) 4418-29.

[60] J. Mowers, M. Uhm, S.M. Reilly, J. Simon, D. Leto, S.H. Chiang, L. Chang, A.R. Saltiel, Inflammation produces catecholamine resistance in obesity via activation of PDE3B by the protein kinases IKKepsilon and TBK1, eLife 2 (2013) e01119.

[61] Y. Zhang, H. Guan, J. Li, Z. Fang, W. Chen, F. Li, Amlexanox Suppresses Osteoclastogenesis and Prevents Ovariectomy-Induced Bone Loss, Scientific reports 5 (2015) 13575. 
[62] K.T. Homan, E. Wu, A. Cannavo, W.J. Koch, J.J. Tesmer, Identification and characterization of amlexanox as a G protein-coupled receptor kinase 5 inhibitor, Molecules 19(10) (2014) 1693749.

[63] C.H. Park, J.H. Lee, M.Y. Lee, J.H. Lee, B.H. Lee, K.S. Oh, A novel role of G protein-coupled receptor kinase 5 in urotensin II-stimulated cellular hypertrophy in H9c2UT cells, Molecular and cellular biochemistry 422(1-2) (2016) 151-160.

[64] C.C. Cho, R.H. Chou, C. Yu, Amlexanox Blocks the Interaction between S100A4 and Epidermal Growth Factor and Inhibits Cell Proliferation, PLoS One 11(8) (2016) e0161663.

[65] S.G. Rani, S.K. Mohan, C. Yu, Molecular level interactions of S100A13 with amlexanox: inhibitor for formation of the multiprotein complex in the nonclassical pathway of acidic fibroblast growth factor, Biochemistry 49(11) (2010) 2585-92.

[66] L.A. Cerezo, M. Remakova, M. Tomcik, S. Gay, M. Neidhart, E. Lukanidin, K. Pavelka, M. Grigorian, J. Vencovsky, L. Senolt, The metastasis-associated protein S100A4 promotes the inflammatory response of mononuclear cells via the TLR4 signalling pathway in rheumatoid arthritis, Rheumatology 53(8) (2014) 1520-6.

[67] P. Norman, Selective JAK inhibitors in development for rheumatoid arthritis, Expert opinion on investigational drugs 23(8) (2014) 1067-77.

[68] Z.J. Tian, W. An, ERK1/2 contributes negative regulation to STAT3 activity in HSS-transfected HepG2 cells, Cell Res 14(2) (2004) 141-7.

[69] A. Douros, K. Grabowski, R. Stahlmann, Safety issues and drug-drug interactions with commonly used quinolones, Expert opinion on drug metabolism \& toxicology 11(1) (2015) 25-39. [70] R. Stahlmann, H.M. Lode, Risks associated with the therapeutic use of fluoroquinolones, Expert opinion on drug safety 12(4) (2013) 497-505.

[71] P. Miotto, D.M. Cirillo, G.B. Migliori, Drug resistance in Mycobacterium tuberculosis: molecular mechanisms challenging fluoroquinolones and pyrazinamide effectiveness, Chest 147(4) (2015) 1135-43. 
TABLE 1. List of signalling molecules detected by RPPA.

\begin{tabular}{|c|c|c|c|}
\hline NF-кB Pathway & PI3K/AKT Pathway & MAPK Pathway & JAK/STAT3 \\
\hline $\begin{array}{c}\text { TRAF2 } \\
\text { p-RIP2(S176) } \\
\text { p-IKB alpha } \\
\text { p-NFKBp65 } \\
\text { A20/TNFAIP3 } \\
\text { c-IAP1 }\end{array}$ & $\begin{array}{c}\text { c-Raf } \\
\text { p-c-Raf } \\
\text { GSK3-B } \\
\text { p-GSK3-B } \\
\text { PTEN } \\
\text { p-PTEN } \\
\text { AKT } \\
\text { p-AKT Serine } \\
\text { p-AKT Threonine } \\
\text { p-BAD 136 } \\
\text { p-PI3k } \\
\text { p-PDK1 }\end{array}$ & $\begin{array}{c}\text { P38 MAPK } \\
\text { p-P38 MAPK (T180/Y182) } \\
\text { p-Sek1/MKK4 (Thr261) } \\
\text { p-HSP27(Ser82) } \\
\text { p-MAPKAPK-2(Thr334) (27B7) } \\
\text { Ask1 } \\
\text { TAK1 } \\
\text { p-MKK3(Ser189)/MKK6(Ser207) } \\
\text { ERK1/2 } \\
\text { p-ERK1/2 } \\
\text { p-SAPK/JNK(Thr) } \\
\text { p-c-Jun (S63) } \\
\text { p-Ask-1 (S83) } \\
\text { 14-3-3-epsilon }\end{array}$ & $\begin{array}{c}\text { STAT3 } \\
\text { p-STAT3 (Tyr705) } \\
\text { p-ELK1 } \\
\text { SOCS3 } \\
\text { JAK2 } \\
\text { p-JAK2 (Tyr221) } \\
\text { p-ATF-2 (Thr71) } \\
\text { c-FLIP }\end{array}$ \\
\hline
\end{tabular}


TABLE 2. SSMD scores calculated for the effects of 80 kinase inhibitors in the BioMol set of compounds on 40 signalling molecules in SK-Hep-1 cells transfected with C33Y-TNFR1 or WTTNFR1.

\begin{tabular}{|l|l|l|l|l|l|}
\hline SSMD* relevance & SSMD score & SK-Hep1-C33Y & Raw hit \% & SK-Hep1-WT & Raw Hit \% \\
\hline Extremely strong & SSMD* $^{*} 5$ & 19 & $0.198 \%$ & 2 & $0.021 \%$ \\
\hline Very Strong & SSMD $^{*}>3$ & 36 & $0.375 \%$ & 22 & $0.229 \%$ \\
\hline Strong & SSMD $^{*}>2$ & 111 & $1.156 \%$ & 227 & $2.365 \%$ \\
\hline Strong & SSMD $^{*}<-2$ & 221 & $2.302 \%$ & 109 & $1.135 \%$ \\
\hline Very Strong & SSMD $^{*}<-3$ & 112 & $1.167 \%$ & 44 & $0.458 \%$ \\
\hline Extremely strong & SSMD $^{*}<-5$ & 29 & $0.302 \%$ & 1 & $0.010 \%$ \\
\hline totals & & 528 & $5.500 \%$ & 405 & $4.219 \%$ \\
\hline
\end{tabular}

TOTAL SSMDs calculated: 9600 per cell type, 19,200 in total. SSMD* relevance guide taken from Refs $1 \& 2$ 
TABLE 3. Drug/concentration combinations ranked 1-35 (out of $>5,000$ ranks) by screening for effects on signalling molecules in C33Y-TNFR1-transfected SK-Hep-1 cells compared to WTTNFR1-transfected SK-Hep-1 cells. Compounds selected for further study are shown in bold.

\section{RANK DRUG: CONCENTRATION}

1___PIROMIDIC ACID: 100

$2 \_$CEFAMANDOLE NAFATE:100

3 CEFUROXIME SODIUM:100

4__CARPROFEN: 10

5__PRALIDOXIME MESYLATE: 100

6 ___ESTRIOL BENZYL ETHER:10000

7 ESTRADIOL-3-SULFATE:100

8 LOMEFLOXACIN HYDROCHLORIDE:100

9 ___METAMPICILLIN SODIUM:100

10__ESTRIOL BENZYL ETHER:100

11 5-FLUORO-5'-DEOXYURIDINE:100

12 FOSFOMYCIN:100

13__CEFAMANDOLE NAFATE: 10000

14___KETOPROFEN:100

15___ESTRADIOL ACETATE:100

16___METHYLERGONOVINE MALEATE:10000

17 RETINOL:100

18__PINDOLOL:100

19___OFLOXACIN:100

$20 \_$TOLFENAMIC ACID: 100

21 IODIPAMIDE: 10000

22 PIROMIDIC ACID:10

23__ MEFENAMIC ACID:100

24 _BUTAMBEN:10000

25 FENSPIRIDE HYDROCHLORIDE:100

26 EPHEDRINE (1R,2S) HYDROCHLORIDE:100

27___DIETHYLTOLUAMIDE:1000

28__CARPROFEN: 100

29__ HECOGENIN: 10000

$30 \_$AMLEXANOX:100

31___GALANTHAMINE HYDROBROMIDE:10000

32___METHYLPREDNISOLONE:100

33__AMINOLEVULINIC ACID HYDROCHLORIDE:100

$34 \quad$ LAPACHOL:100

35 ERGOCALCIFEROL:10000
SCORE NATURE / FUNCTION

\begin{tabular}{|c|c|}
\hline 11.40 & Quinolone antibiotic \\
\hline 13.71 & Cephalosporin antibiotic \\
\hline 15.52 & Cephalosporin antibiotic \\
\hline 16.15 & COX inhibitor / NSAID \\
\hline 17.40 & Antidote for organophosphate poisoning \\
\hline 18.21 & Oestrogen steroid hormone \\
\hline 18.79 & Oestrogen steroid hormone \\
\hline 19.10 & Fluoroquinolone antibiotic \\
\hline 19.65 & $\beta$-lactam antibiotic \\
\hline 20.52 & Oestrogen steroid hormone \\
\hline 20.62 & Inhibitor of thymidine synthesis \\
\hline 21.85 & Phosphonic acid antibiotic \\
\hline 22.10 & Cephalosporin antibiotic \\
\hline 22.29 & COX inhibitor / NSAID \\
\hline 22.30 & Oestrogen steroid hormone \\
\hline 23.22 & Inducer of smooth muscle contraction \\
\hline 24.47 & Vitamin A \\
\hline 24.59 & $\beta$-Adrenoceptor antagonist \\
\hline 24.92 & Fluoroquinolone antibiotic \\
\hline 26.31 & COX inhibitor / NSAID \\
\hline 26.40 & Radiopaque contrast medium \\
\hline 26.71 & Quinolone antibiotic \\
\hline 27.18 & COX inhibitor / NSAID \\
\hline 27.92 & Anaesthetic \\
\hline 28.76 & Antitussive / anti-inflammatory \\
\hline 28.89 & $\beta$-Adrenoceptor agonist / bronchodilator \\
\hline 29.13 & Insect repellant \\
\hline 29.70 & COX inhibitor / NSAID \\
\hline 29.71 & Steroid saponin \\
\hline 30.05 & Anti-inflammatory/anti-allergic agent \\
\hline 30.35 & Alkaloid used in dementia treatment \\
\hline 30.40 & Anti-inflammatory glucocorticoid \\
\hline 30.80 & Porphyrin precursor for photodynamic therapy \\
\hline 30.89 & Vitamin $\mathrm{K}$ derivative \\
\hline 30.97 & Vitamin $\mathrm{D}_{2}$ \\
\hline
\end{tabular}


TABLE 4. Summary of all instances in which increasing concentrations of the lead drugs significantly ( $\mathrm{p} \leq 0.1$; precise $\mathrm{p}$ values given in brackets) reduced $(\downarrow)$ or increased ( 4 ) expression of signalling molecules in PBMCs from C33Y-TNFR1 TRAPS patients towards levels seen in PBMCs from normal healthy control subjects.

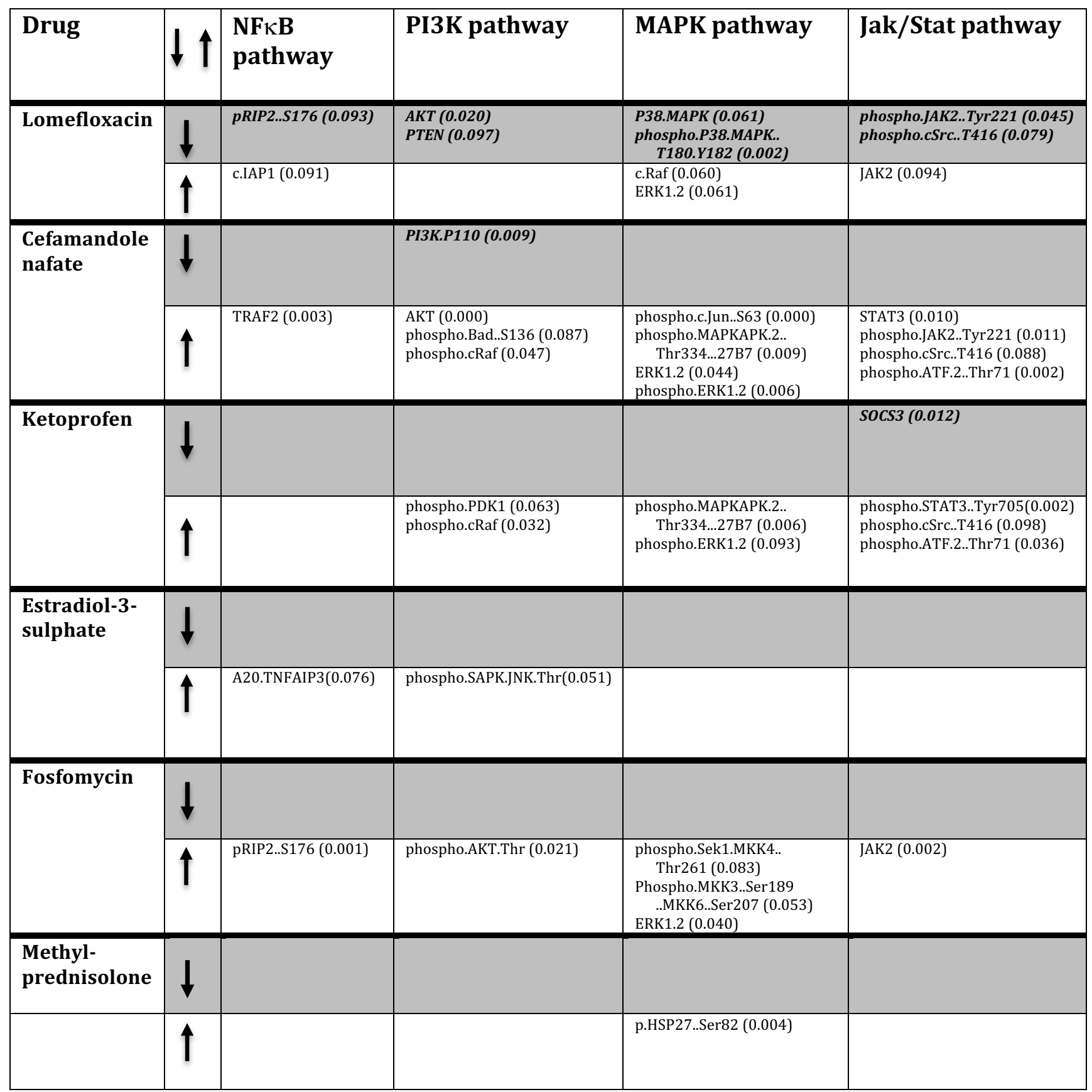




\section{FIGURE LEGENDS}

Figure 1. (A) Coefficient of variation calculated for BCA protein yield and MTT cell viability for row, column and entire 96 well plates where SK-Hep-1 cells have been robotically seeded at 100,000 cells/well. Cells were seeded, settled overnight at $37^{\circ} \mathrm{C}, 5 \% \mathrm{CO}_{2}$ and then exchanged to serum-free medium for 24 hours, followed by robotic medium removal, washing and handling for lysis and subsequent BCA/MTT assay. A box and whisker plot is shown, with median represented by a black line within the box that represents the interquartile range, using Tukey's estimation for whisker length. The mean is represented by a '+' symbol. Outliers are plotted as black circles. (B) Box plots, showing median and interquartile range and whiskers (Tukey's estimation) of actin signal levels from RPPA analysis of triplicated SK-Hep-1 seeded plates (10 cells/well). Duplicate array features were printed per sample (well) onto separate arrays and probed for a standardly used housekeeping protein ( $\beta$-actin) and four alternatives ( $\alpha$-tubulin, GAPDH, NuP98 and $\beta$-catenin). A unique sample ( $n=96)$ was used for each experiment. Mean $\mathrm{CV} \pm \mathrm{SD}$ of the 3 replicates is shown above each group of plots (AFU, arbitrary fluorescence units). (C) Box plots, showing median and interquartile range and whiskers (Tukey's estimation), of the coefficient of variation of actin signal levels from RPPA analysis of SK-Hep-1 seeded plates $\left(10^{5}\right.$ cells/well). Replicate wells were subjected to 14 different treatments but, for this analysis, the data were used to determine reproducibility of printing. Duplicate array features were printed per sample (well) onto separate arrays and probed for $\beta$-actin. For DMSO $( \pm)$ and PMA treatment, $\mathrm{n}=16$ biological replicates; for all other treatments, $\mathrm{n}=32$ biological replicates. CVs were calculated for each replicate group on each array $(n=11)$. Both technical replicates for a sample were excluded from analysis if one or both were flagged for poor spot morphology or other imaging issues during scanning, or if the actin signal for at least one replicate was identified as $>5.5$ SD away ( \pm ) from the mean signal of the replicate set. (D) (i) SK-Hep-1 cells response to 100nM PMA stimulation over 1 hour as measured by increased phosphorylation of HSP-27. Z' = 0.368 and SSMD $\beta=6.68$ averaged for this set of assays, $n=24$ replicates. (ii) Upstream inhibition of PMA-induced ERK1/2 phosphorylation by the MEK1/2 inhibitor PD98059 (IC $50 \sim 5-10 \mu \mathrm{M}$ ). For range QC, the Z' was calculated to be 0.33 and the $\operatorname{SSMD} \beta=6.16(\mathrm{~N}=6)$. (E) Examination of the action of three inhibitors (applied at 7 concentrations followed by PMA stimulation for 1 hour) upon 14 intra-cellular signalling intermediates. (i) PD98059, a MEK1/2 inhibitor (IC50 5$10 \mu \mathrm{M}$ ), showing expected effects on phosphorylation of ERK1/2 (pERK1/2, green circles, SSMD $\beta$ score $=8.58$ ) and effects on MEK $1 / 2$ abundance (orange squares, SSMD $\beta$ score $=4.8$ ). (ii) 
FR180204 is an ERK1/2 inhibitor that does not stop ERK1/2 phosphorylation and, as expected, shows no effect on the molecules tested. (iii) SB203580 is a MAPKAPK2 phosphorylation inhibitor; however, an inhibitory effect on pERK1/2 is observed (green circles, SSMD $\beta$ score = 3.81). All sample time points are from single well cultures, examined as 7 technical replicates on the 14 arrays used.

Figure 2. (A) representative example of a single array, printed using a $2 x 4$ pin arrangement. Not all space on the array is used at this feature spacing. (A) also shows the dimensions and layout of the array. (B) an array after probing and scanning for $\beta$-actin (false-coloured red) and phosphoAKT (Threonine) (false-coloured green) with appropriate antibodies. Each chemically treated culture well is represented as duplicate features. The array holds all three experimental replicates and all controls.

Figure 3. Analysis of compound activity in screening of NINDS and Sequoia compound libraries for effects on signalling molecules in C33Y-TNFR1-transfected SK-Hep-1 cells compared to WTTNFR1-transfected SK-Hep-1 cells. (a) Cumulative profile of scores (expressed as binned $\log _{10}$ values) for all drugs at all concentrations: red bars $-10 \mathrm{~nm}$; orange bars $-10^{2} \mathrm{~nm}$; yellow bars $10^{3} \mathrm{~nm}$; green bars $-10^{4} \mathrm{~nm}$. Lower scores (to the left of the distribution) correspond to higher suppressive effects on the C33Y-TNFR1 signalome. (b) Clustering of the screened library compounds into structural families with the number of compounds in each cluster ( $\mathrm{x}$-axis) plotted against the mean logged score for each cluster in suppressing the C33Y-TNFR1 signalome (y-axis). Lower scores correspond to cluster with higher suppressive activity. Compound clusters of interest based on cluster size and mean score are highlighted. (c) Identification of compounds predicted to show suppressive activity on the C33Y-TNFR1 signalome from amongst untested compounds listed in ChEMBL as being in phase IV clinical trials with molecular weights between 100 and 600; these predictions were generated by the application to the ChEMBL data set of a Bayesian model based on the compound activity scores derived from screening the NINDS and Sequoia libraries on SK-HEP-1 TNFR1-tranfectants. The ChEMBL compounds predicted to be active are those with a score $\geq 10$ in the Bayesian model (which relates to the probability of being active in the microarray assay), and are: light blue quinolones/fluoroquinolones/quinolinones; dark blue - cephalosporins; pink - estradiols; orange - glucocorticoid steroids; red - other steroids. Compounds shown as inverted triangles fail 'Lipinski's rule of five' and so are unlikely to serve as orally active drugs in humans. 
Figure 4. Summary of the modulatory effects of lomefloxacin on pro-inflammatory signalling pathways in TRAPS patients' PBMCs. The expression of molecules shown in red was dosedependently down-regulated by lomefloxacin, those in green were upregulated, and those in blue were unaffected. 
Figure 1.

A

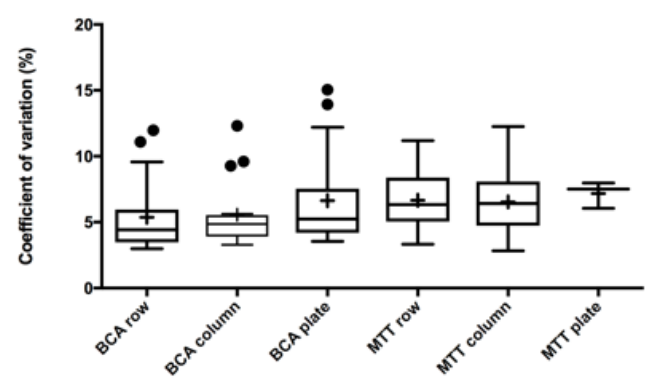

B

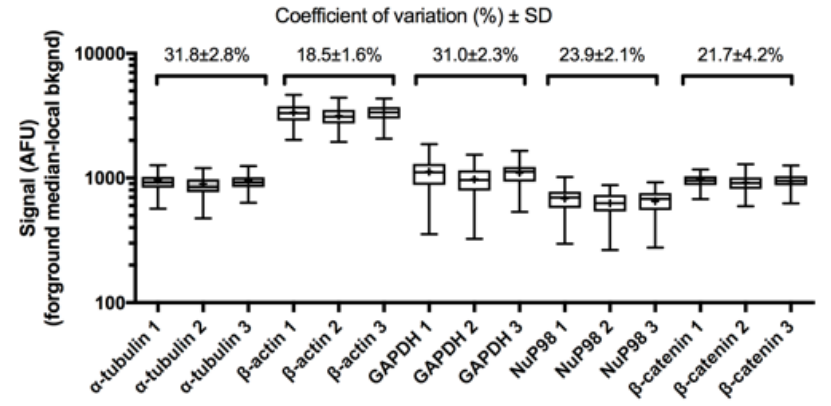

$A$

(i) pHSP27-PMA time course

D

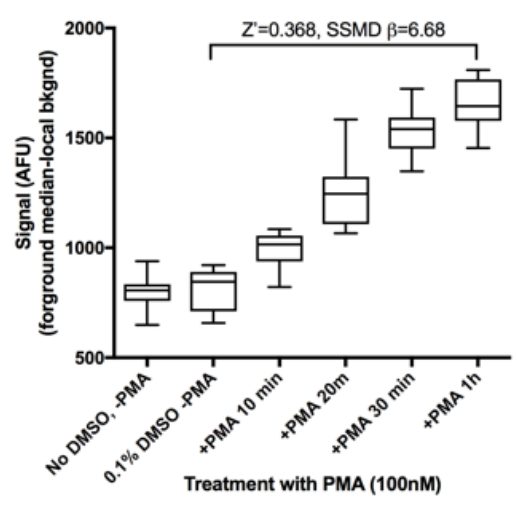

(ii) PERK1/2 - PD98059 effects

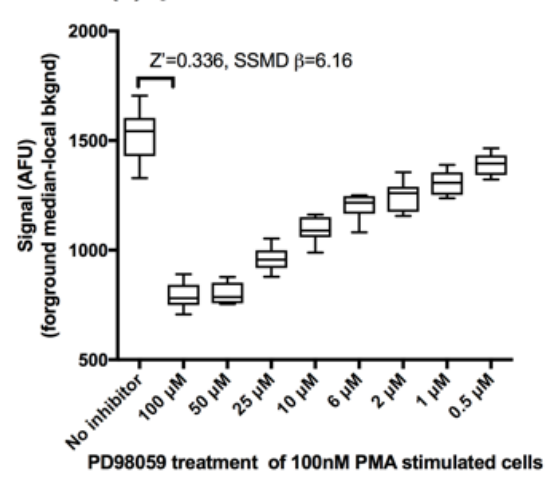

$E(i)$

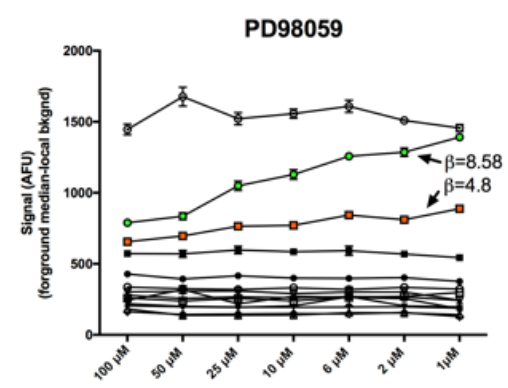

E(ii)

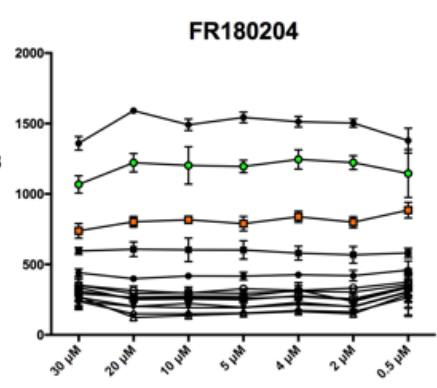

E(iii)

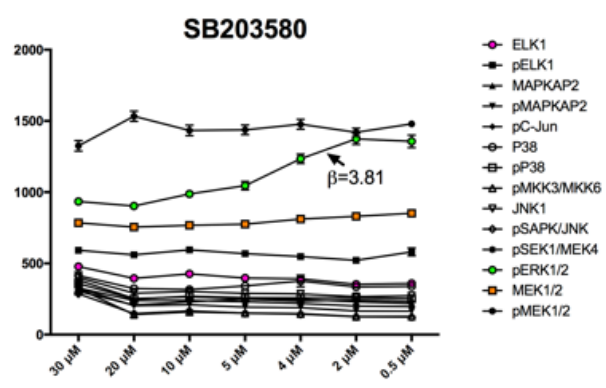


Figure 2.

A

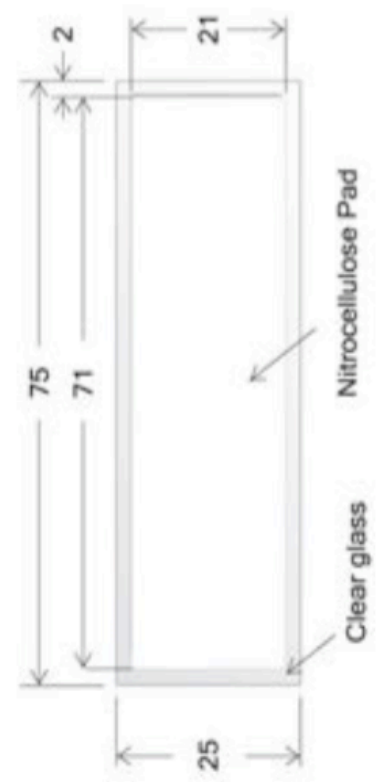

B

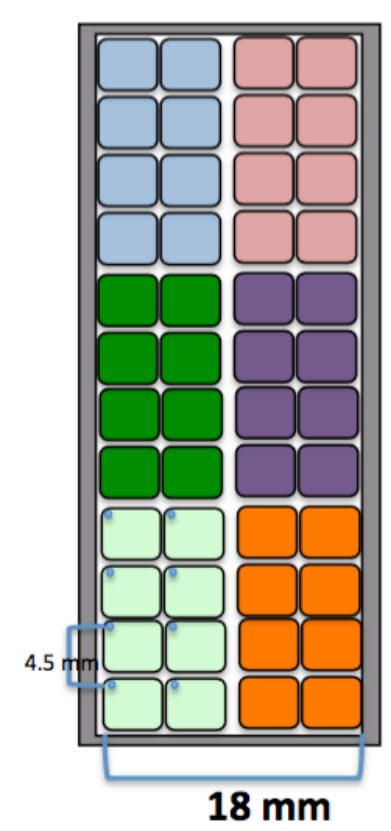

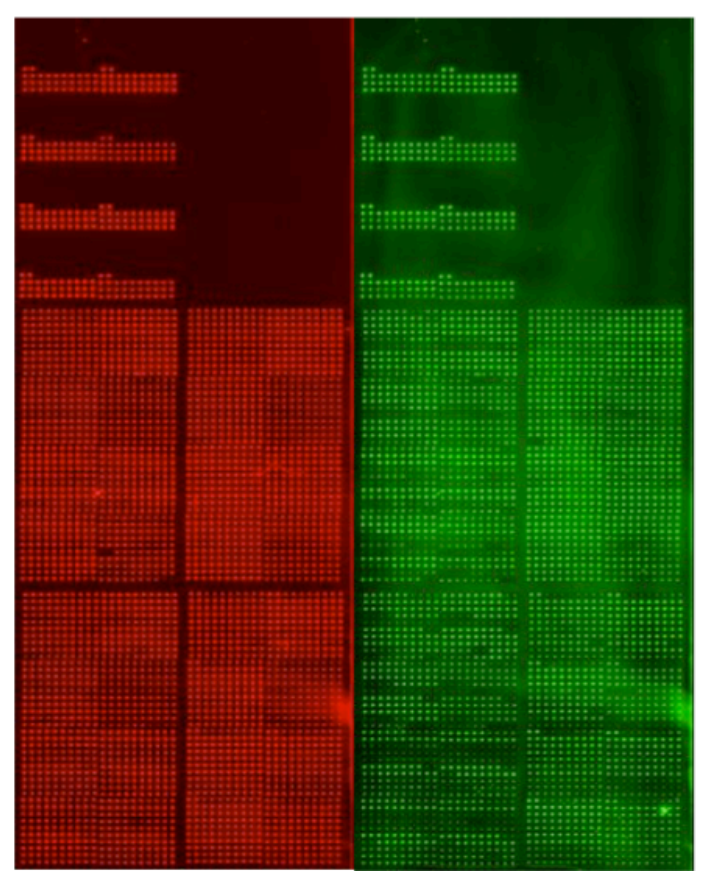

B-actin pAKT Threonine 


\section{Figure 3}

(a)

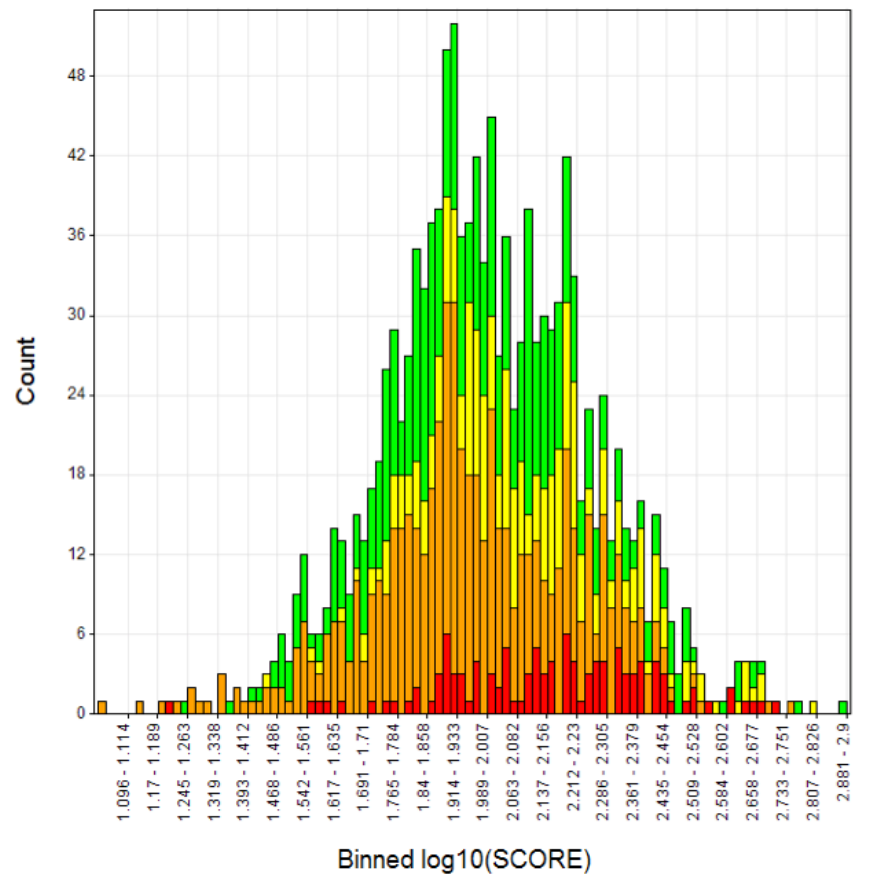

(c)

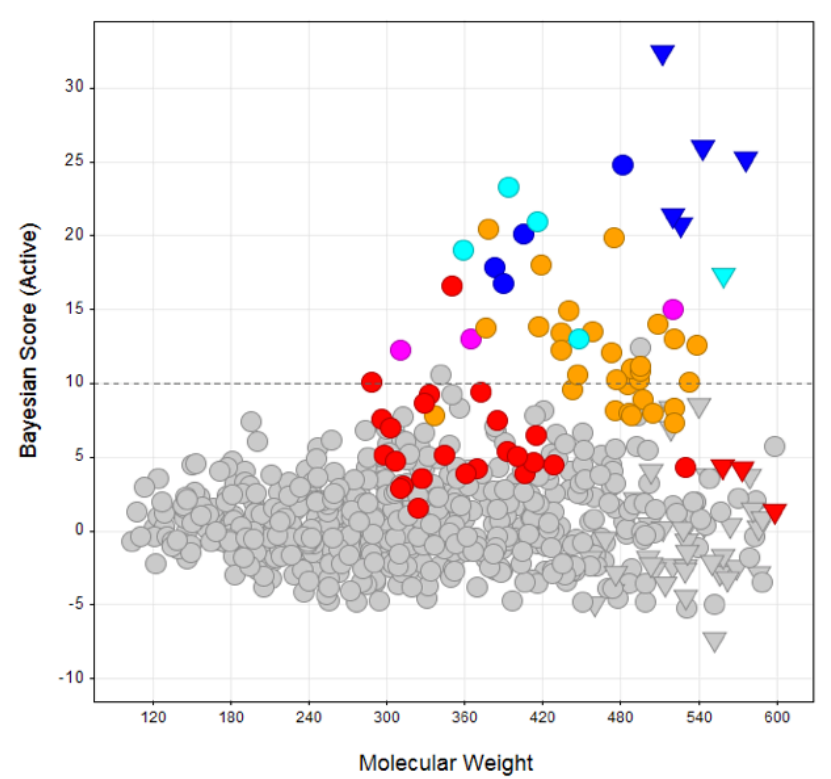

(b)

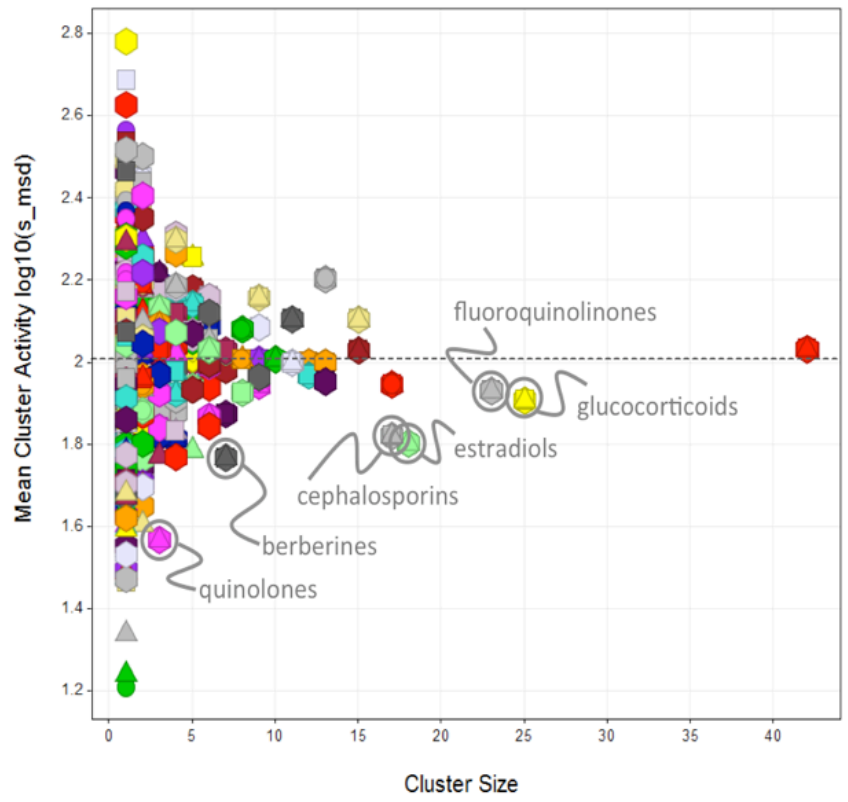


Figure 4

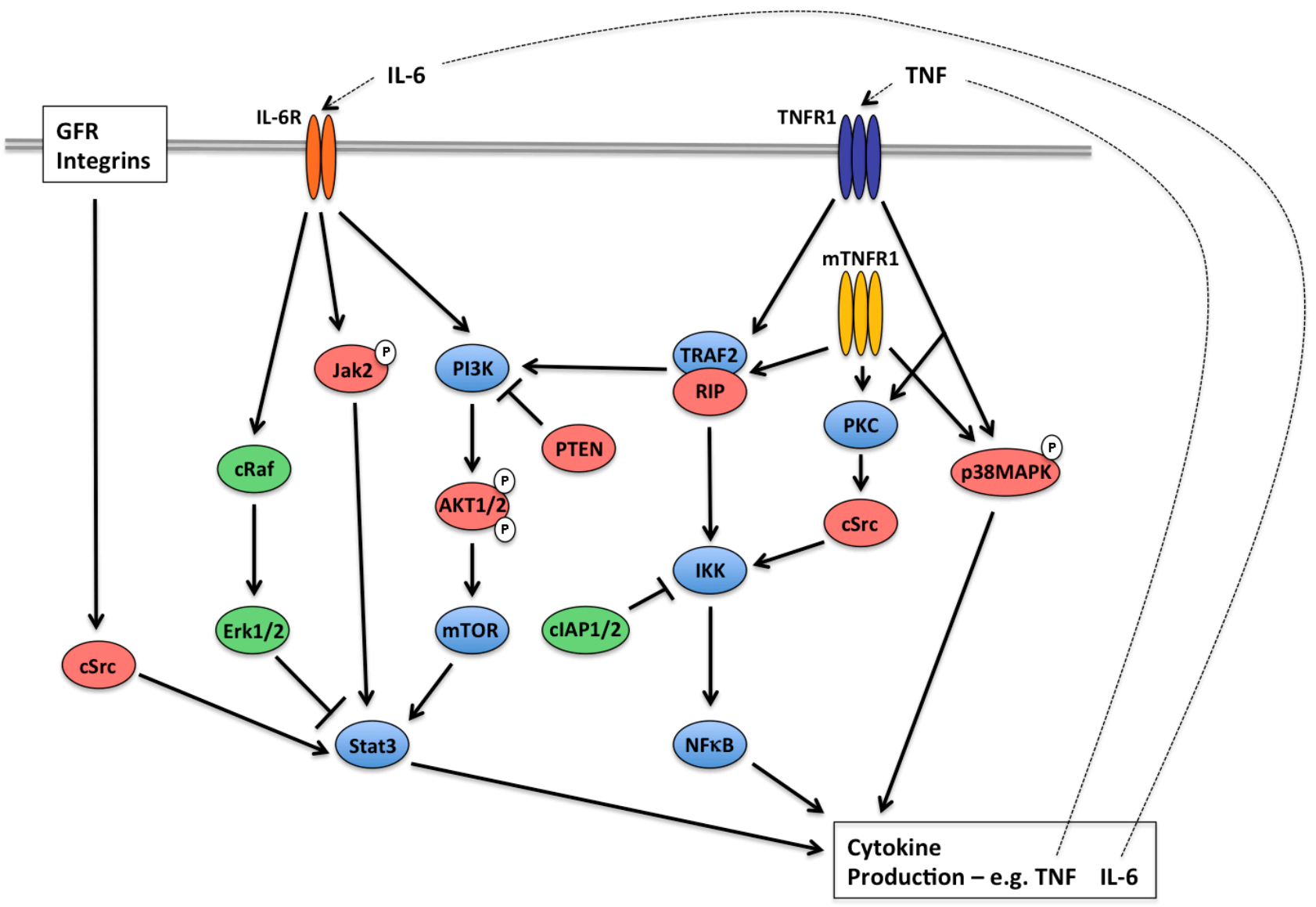




\section{Appendix A. Supplementary Information}

\section{A signalome screening approach in the autoinflammatory disease TNF Receptor Associated Periodic Syndrome (TRAPS) highlights the anti-inflammatory properties of drugs for repurposing}

Ian Todda, ${ }^{a}$, Ola H. Negm ${ }^{\mathrm{a}, \mathrm{b}, \mathrm{g}, 1}$, Jenna Reps ${ }^{\mathrm{c}, \mathrm{h}}$, Paul Radforda, Grazziela Figueredo ${ }^{c}$, Elizabeth M. McDermottd ${ }^{d}$ Elizabeth Drewe ${ }^{d}$, Richard J. Powella, Susan Bainbridgea, Mohamed Hameda, Sharon Crouche, Jon Garibaldic, Steve St-Gallayf, Lucy C. Fairclougha, ${ }^{a, 2}$, Patrick J. Tighea,2 aSchool of Life Sciences, The University of Nottingham, A Floor West Block, Queen's Medical Centre, Nottingham, NG7 2UH, UK

${ }^{b}$ Medical Microbiology and Immunology Department, Faculty of Medicine, Mansoura University, Egypt;

cAdvanced Data Analysis Centre, School of Computer Science, The University of Nottingham, Jubilee Campus, Nottingham, NG8 1BB, UK;

${ }^{d}$ Nottingham University Hospitals National Health Service Trust, Queen's Medical Centre Campus, Nottingham, NG7 2UH, UK;

${ }^{e}$ Business Engagement and Innovation Services, The University of Nottingham, Jubilee Campus, Nottingham, NG8 1BB, UK;

fSygnature Discovery Limited, BioCity, Pennyfoot Street, Nottingham, NG1 1GF, UK

\section{Mathematical data analysis methodology}

Step 1

\section{1a) Data Creation}

Considering 1360 drugs, a wild type sample and C33Y mutation sample are given the drug at 4 different concentrations. There are also 16 control wild type and 16 C33Y mutation samples given the DMSO solution at 4 different concentrations. This 
leads to $11,008=[(1360+16) \times 4 \times 2]$ data points. For each data point, $\mathrm{x}$, we have the sample type (C33Y or WT), the drug, the concentration of the drug and the signals obtained via the microarray for 40 various molecules.

Let $x_{i j k}^{n}$ correspond to molecule n's signal for sample $i \in\{0,1\}$, drug $j \in \mathbb{Z}_{\leq 1376}$ and concentration $k \in \mathbb{Z}_{\leq 4}$. Where $i=0$ when the sample is a C33Y mutation and $i=1$ when the sample is a WT, $j \in[1361,1376]$ for the DMSO 1-16 samples and 1 to 1360 for the other drug samples and $k$ is the log to the base 10 of the samples drug concentration. Then $X^{n}=\left\{x_{i j k}^{n}\right\}$ is the set of all the signals returned by the microarray for molecule $n$, where $n \in \mathbb{Z}_{\leq 40}$.

We calculate the distance between the signal intensities for samples corresponding to C33Y given drug $i$ and the samples corresponding to WT with no drug $(j \in[1361,1376])$ at concentration $\mathrm{k}$ as,

$d_{i k}=\sum_{n} \frac{\left(x_{0 i k}^{n}-\operatorname{Median}\left(\left\{x_{1361 i k}^{n}, x_{1362 i k}^{n}, \ldots, x_{1376 i k}^{n}\right\}\right)\right)}{\operatorname{IQR}\left(\left\{x_{1361 i k}^{n}, x_{1362 i k}^{n}, \ldots, x_{1376 i k}^{n}\right\}\right)}$

Where, for each antibody, this calculates the difference between the signal for the C33Y given drug $i$ at concentration $k$ minus the median signal for the WT given DMSO at concentration $k$ divided by the inter quartile range (IQR) of the signal for the WT given DMSO at concentration $k$. This is effectively a weighted sum, where more emphasis is added to the drug and concentration pairs that appear to have less signal variance in the wild type patients. The sum over all the molecules gives the overall measure of difference between the C33Y TRAPs patient given drug $i$ at concentration $k$ and the WT patient given no drug. Finally, we find the best concentration for drug $i$ by finding, $\underset{k}{\operatorname{argmin}}\left\{d_{i k}\right\}$.

We then return the rank of drug $i$ and concentration $k$ in ascending order of the measure $d_{i k}$. Clinical experts reviewed the ranked list and chose 7 drugs to be investigated in more detail based on the rank. How were step 1 results used to identify the drugs? 
Step 2

\section{2a) Data creation}

For step 2, as in step 1, we had the 40 molecule signals at four concentrations of the drugs of interest. The calculations are exemplified here for 5 C33Y TRAPs patients and 4 WT healthy control subjects without the mutation for each molecule and concentration.

\section{2b) Preprocessing}

After normalizing the micro array data we removed any potential signal outliers. For each set of C33Y patients or each set of WT patients we calculated the median absolute deviation (MAD) for each drug and concentration combination. Any signal that was three times the MAD away from the median signal of the group was removed.

\section{2c) Investigating interaction importance}

To model the data (with outliers removed), we applied mixed-effect models of the form:

$y_{i j}=X_{\mathrm{i}} \beta+Z_{j} b_{j}+\varepsilon_{i}$

$\boldsymbol{b}_{\boldsymbol{j}} \sim \mathbf{N}(\mathbf{0}, \boldsymbol{\psi})$

$\varepsilon_{i} \sim \mathbf{N}\left(\mathbf{0}, \sigma^{2} \Lambda_{i}\right)$

Where $\boldsymbol{y}_{i j} \in \mathbb{R}^{\mathbf{2 8 \times 9}}$ is the signals observed for the 9 patients, taking 7 different drugs at 4 different concentrations, $\mathbf{X}_{\mathbf{i}} \in \mathbb{R}^{\mathbf{2 8} \times \boldsymbol{p}}$ is the matrix of fixed effects, $\boldsymbol{\beta} \in$ $\mathbb{R}^{9 \times \boldsymbol{p}}$ is the vector of fixed effect coefficients, $Z_{j} \in \mathbb{R}^{9 \times 1}$ is the random effects, $b_{j} \in$ $\mathbb{R}$ is the random effects coefficient (per patient random effect), $\varepsilon_{i}$ is the $252 \times 1$ vector of errors for observations, $\psi$ is the $\mathrm{q} \times \mathrm{q}$ covariance matrix for the random effects and $\sigma^{2} \Lambda_{i}$ is the $4 \times 4$ covariance matrix for the errors.

For each drug and molecule, the two mixed-effect models applied were,

- Model 1: 


$$
\begin{aligned}
y_{i j}=\beta_{1}+ & \beta_{2} \text { drug }_{i}+\beta_{3} \text { mut }_{i}+\beta_{4} \text { conc }_{i}+\beta_{5} \text { drug }_{i} \text { mut }_{i}+ \\
& \beta_{6} \text { drug }_{i} \text { conc }_{i}+\beta_{7} \text { mut }_{i} \text { conc }_{i}+\beta_{8} \text { drug }_{i} \text { mut }_{i} \operatorname{conc}_{i}+ \\
& b_{j}+\varepsilon_{i}
\end{aligned}
$$

- Model 2:

$$
\begin{aligned}
y_{i j}=\beta_{1}+ & \beta_{2} \text { drug }_{i}+\beta_{3} \text { mut }_{i}+\beta_{4} \text { conc }_{i}+\beta_{5} \text { drug }_{i} \operatorname{conc}_{i} \\
& +\beta_{6} \text { mut }_{i} \operatorname{conc}_{i}+b_{j}+\varepsilon_{i}
\end{aligned}
$$

where $\boldsymbol{y}_{\boldsymbol{i}}$ is the $\mathrm{i}^{\text {th }}$ signal reading of the molecule for patient $\mathrm{j}, \boldsymbol{d r} \boldsymbol{u} \boldsymbol{g}_{\boldsymbol{i}}$ is an indicator of whether the drug of interest was given to the patient $j$ for the ith signal reading, $\boldsymbol{m u} \boldsymbol{t}_{\boldsymbol{i}}$ is an indicator of whether the patient $\mathrm{j}$ had the TRAPs mutation and $\boldsymbol{c o n c}_{\boldsymbol{i}}$ is the $\log 10$ of the concentration of the drug given for the ith signal reading of patient $j$. The random effects term $\boldsymbol{b}_{\boldsymbol{j}}$ means than patient variation is included into the models, with the random effect coming from a Normal distribution with 0 mean. The second equation excludes both the interaction between the drug and mutation and the interaction between concentration, mutation and drug. We then applied a log likelihood-ratio test to determine whether the first model (including the additional interactions) significantly improved the model compared to the second model fit while accounting for the additional complexity of the model with the interaction terms. We used a significant level of $10 \%$ due to the small number of samples limiting the power of the analysis.

\section{2d) Alleviating vs exacerbating the difference}

In the previous step we have identified the drugs that potentially cause patients with the TRAPs mutation to have a different molecule signal dynamic compared to patients without the mutation and drug. However, this does not tell us whether the drug exacerbates or alleviates the different in the antibody signal between the patients with TRAPs and those without. We want to find drugs that normalise the TRAP's mutation molecule signals (e.g. make them representative of the signal for wild type patients taking no drug). To investigate this, we also calculated the fixed effect coefficients for model 3 , 


$$
\begin{gathered}
y_{i}=\beta_{0}+\beta_{1} \text { drug: } T_{i} \text { mut: } T_{i} \text { conc }_{i}+\beta_{2} \text { drug: } F_{i} \text { mut: } T_{i} \text { conc }_{i}+ \\
\beta_{3} \text { drug: } T_{i} \text { mut: } F_{i} \text { conc }_{i}+\beta_{4} \text { drug: } F_{i} \text { mut: } F_{i} \text { conc }_{i}+b_{i}+\varepsilon_{i}
\end{gathered}
$$

We use model 3 to determine whether the coefficient of the fixed effect corresponding to taking the drug when the patient had the mutation $\left(\boldsymbol{\beta}_{\mathbf{1}}\right)$ was less than the coefficient of the fixed effect corresponding to not taking the drug when the patient did not have the mutation $\left(\boldsymbol{\beta}_{4}\right)$. Justification of this is that the patients with the mutation have an elevated signal for the molecules investigated, so if the presence of the drug and mutation interaction is causing the signal to decrease at a greater rate (or increase at a slower rate) than the absence of the drug and mutation interaction over concentration, then this suggests the TRAPs patients' signals are normalizing as the drugs concentration increases.

\section{2e) Combining results}

Finally, we calculate the number of molecule for each drug where the likelihood test showed a significant interaction between the drug, concentration and mutation (model 1 fit the data better than model 2) and the drug showed signs of normalizing the molecule's signal (coefficient $\boldsymbol{\beta}_{\mathbf{1}}$ was less than $\boldsymbol{\beta}_{\mathbf{4}}$ in model 3). 